\title{
Planning of Cascade Hydropower Stations with the Consideration of Long-Term Operations under Uncertainties
}

\author{
Changjun Wang $\mathbb{D}^{1}$ and Shutong Chen $\mathbb{D}^{1,2}$ \\ ${ }^{1}$ Glorious Sun School of Business and Management, Donghua University, Shanghai 200051, China \\ ${ }^{2}$ Department of Industrial and Systems Engineering, Virginia Tech, Blacksburg, VA 24061, USA \\ Correspondence should be addressed to Changjun Wang; cjwang@dhu.edu.cn
}

Received 8 July 2019; Revised 21 September 2019; Accepted 8 October 2019; Published 28 November 2019

Guest Editor: Chun Wei

Copyright (c) 2019 Changjun Wang and Shutong Chen. This is an open access article distributed under the Creative Commons Attribution License, which permits unrestricted use, distribution, and reproduction in any medium, provided the original work is properly cited.

\begin{abstract}
In the location-related planning of a hydropower system, the consideration of future operations under uncertainties can make the decisions sustainable and robust. Then, it is of great importance to develop an effective approach that deals with the longterm stochasticity due to the long-lasting effects of the location selections. Thus, we propose a multistage stochastic programming model to optimize the planning decisions of cascade hydropower stations and the long-term stochastic operations in an integrated way. The first stage (i.e., the planning stage) in the model deals with the location and capacity decisions of the hydropower stations, while the subsequent stages implement the scheduling decisions under each stagewise stochastic scenario. To address the curse of dimensionality caused by the long-term stochastic operations, we further propose a novel dimensionality reduction approach based on dual equilibrium to transform the multistage model into a tractable two-stage stochastic program. The applicability of our approach is validated by a case study based on a basin of Yangtze River, China, and corresponding sensitivity analysis.
\end{abstract}

\section{Introduction}

Given a basin, the hydropower stations to-be-built usually could be multiple. Even only a hydropower station in the current timetable, it is still necessary to consider the impacts of other possible hydropower stations in the future because of their interdependence. Moreover, such a plan that involves major capital investments must be made for a longstanding future. Hence, subsequent operations of all the stations, which influence the effect of the planning decisions, should be considered. Such an integrated way can help to get the global optimum in disasters mitigation, irrigation water supply, and clean power production $[1,2]$. However, in the long-term operations, natural or social uncertainties, such as water inflows and demands, are inevitable and influence the performance of the hydropower system significantly [3]. Thus, the problem faced by the decision-maker is long-term stochastic optimization. The "long-term" we mentioned here is far beyond the long-term scale considered in previous works, such as 3-5 years in Gjelsvik et al. [4] or 12-period used in Cheng et al. [5], Wang et al. [6], and Xu and Mei [7], because of the long-lasting effects of the location decisions. Hence, the challenge is how to handle such overlong stochastic nature.

In previous works, the decisions on locations and operations of a hydropower system have been extensively, but separately, studied. Specifically, when a decisionmaker plans a hydropower station, advanced technologies, such as geographical information system (GIS), are often utilized to obtain geological, climatic, and topographical information in most recent studies. Based on this information, different mathematical methods are further utilized for spotting site by synthesizing natural, engineering, and social criteria. For example, Larentis et al. [8] propose a GIS-based DEM (digital elevation model) composed of the preidentification of promising 
sites and the multicriteria feasibility assessment of the final set, in which energy, technical, and environmental factors are considered together. Another GIS-based DEM is proposed by Kusre et al. [9] which use a hydrologic model to assess water resource utilization under different site candidates. Similar works can be referred to Serpoush et al. [10], Zaidi and Khan [11], etc. Besides the above assessment models, the optimization technique becomes another alternative in recent years. For example, Hosnar and Kovač-Kralj [12] identify the optimal installation locations by maximizing an ecoprofit objective at technological, economic, environmental, and social constraints. To identify the appropriate hydropower dam location, Loannidou and O'Hanley [13] develop a mixedinteger linear programming model to optimize the hydropower potential with the consideration of river connectivity. In these studies, the future power generation and hydrologic dynamics are described by the empirical formulas in the cumulative form. Thus, time-varying operations under uncertainties are ignored in the setting of location-related planning.

The optimization of the multistage operational activities, such as water storage, supply, and power generation, is an important issue which has drawn lots of studies. Most of them focus on a given hydropower system involving a single hydropower plant (e.g., Vieira et al. [14]) or multistations. For the multistation operations in the deterministic setting, two main stream of approaches have been applied. At first, to pursue the optimality, mathematical programming methods, especially dynamic programming (DP), are often used but also suffer from the computational challenge due to the number of stages and stations. Thus, Cheng et al. [15] reduce the scale of the problem by limiting their work in the "short-term" horizon. Li et al. [16] consider a decomposition-coordination mechanism to reduce dimensionality. Li et al. [17] and Cheng et al. [5] propose to parallelize the DP algorithm to reduce the computation time. The former uses the distributed memory architecture and the message passing interface protocol, while the latter considers the Fork/Join parallel framework in a multicore environment. Feng et al. [18] and Feng et al. [19] focus on the simplification of state set to accelerate the implementation of DP. Cheng et al. [20] and Feng et al. [21] adopt progressive optimality algorithm to modify the conventional DP by dividing the multistage problem into a sequence of subproblems and thus reduce the computational burden. Second, in order to address the computational complexity, numerous heuristic algorithms have been considered by sacrificing some optimality in recent years. Typical methods used in this stream include particle swarm optimization [22, 23, 24], electromagnetism-like mechanism [25, 26], genetic algorithm [27], water cycle algorithm [7], and artificial intelligence algorithms [28]. However, all these studies focus on the deterministic setting.
Uncertainties extensively exist in hydropower systems. For example, water inflows usually vary and cannot be accurately predicted since long-term meteorological forecasts are unreliable $[29,30]$. Thus, the optimization of hydropower operations under uncertainties is basically a risk-based decision-making problem [31]. The stagewise stochastic process can be represented as a scenario tree; hence, the multistage stochastic programming (MSSP) model, which optimizes the expected value based on the scenario set, has been popularly used. For example, Fleten and Kristoffersen [32] develop an MSSP model to make the decisions on power generation, in which uncertain water inflows and electricity market prices are considered, with the optimization of the expected benefit. Chazarra et al. [33] propose an MSSP model for a hydropower system, taking uncertain water inflows and electricity market prices into account, to simultaneously maximize the expected profit in both energy and regulation reserve markets. To deal with uncertain streamflow, a multiobjective MSSP model is presented by Xu et al. [34] to optimize scheduling strategies. The first objective is to maximize direct revenue from energy production, and the second one is to minimize the expected energy shortfall percentage. Séguin et al. [35] address the stochastic hydropower unit commitment and loading problem under the uncertain inflows. However, all these works only consider the short-term horizon. Thus, off-the-shelf solvers can be applied to solve these models directly.

With the increase of the number of decision stages, the size of the MSSP grows dramatically which often requires decomposition methods [36]. A state-of-the-art one is the stochastic dual DP algorithm which is a sampling-based variant of nested Benders decomposition. Hjelmeland et al. [37] use it to handle a medium-term scheduling issue for a single producer under uncertain inflows and prices within one to three-year horizon. Most related works consider the medium-term setting as Gjelsvik et al. [4]; Helseth et al. [38]; Hjelmeland et al. [39]; and Poorsepahy-Samian et al. [40]. However, as indicated by Hjelmeland et al. [39], although decomposition methods can help to alleviate the solving complexity, the computation would significantly become slow with the increase of system size and decision stages. It shows the difficulty of multistage stochastic optimization with high dimensions of uncertainty.

In summary, a considerable amount of research has been conducted for the hydropower system design and operation. However, to the best of our knowledge, the location decisions in existing studies have not taken multistage operations into account. In terms of operations, numerous studies focus on developing efficient exact or heuristic algorithms for the deterministic setting, whereas many endeavors utilize the MSSP to address the stochastic operations. However, the high dimensionality characteristic has become the main bottleneck limiting the application of optimization approaches [41]. Even in the deterministic setting, the computation of the hydropower 


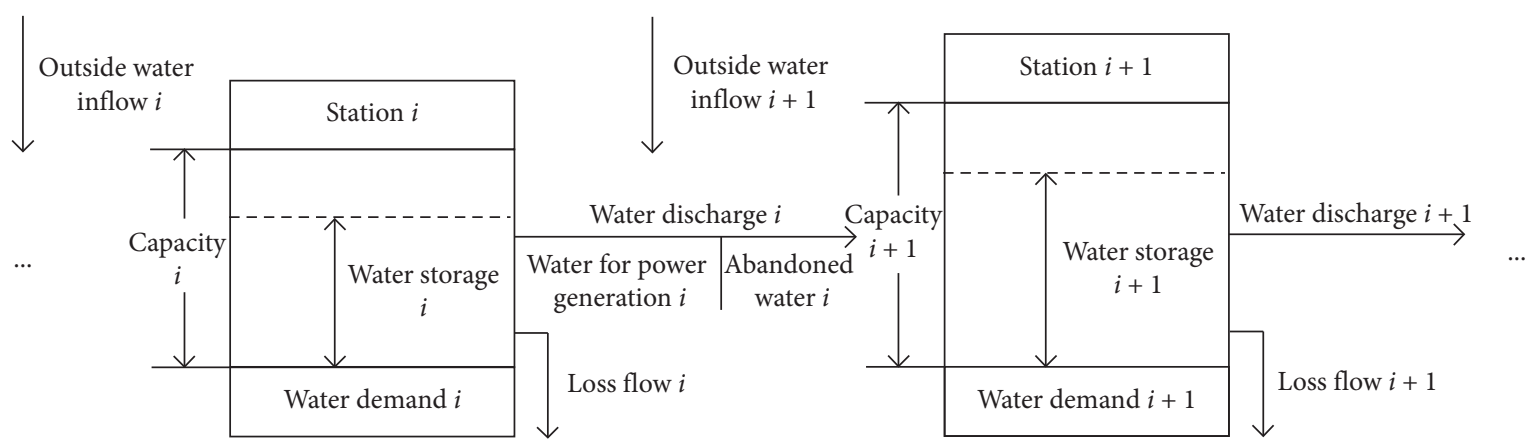

FIGURE 1: Illustration of operational activities.

system operation problem is highly complex when the number of operational stages is big [19]. With the consideration of randomness, the optimization of the MSSP should be implemented on all the stagewise stochastic realizations, which further increase the solving burden. Hence, most existing works only focus on short- or medium-term stochasticity. However, the location selection is the planning decision that involves major capital investments and has an overlong effect. Thus, such a decision should be made from the long-lasting perspective, in which uncertainties would occur inevitably. Hence, the long-term stochasticity should be considered in the hydropower design, which is a difficult task.

We contribute to the existing literature by planning a cascade hydropower system with the consideration of long-term stochastic operations. Most mainstream algorithms, including DP for the deterministic setting and the decomposition methods for the stochastic setting, suffer from the number of stages. Few papers have looked specifically into long-term stochastic models. Considering that the purpose of our work is the location decisions rather than providing accurate schedules, in order to address the dimensionality issue, an intuitive idea is to keep the influence of stochastic operations while reducing the number of stages. Thus, applying the method of dual equilibrium (DE) $[42,43]$, we propose a novel dimensionality reduction approach which aggregates the long-term operational impacts on the present and thus simplifies the MSSP model to a two-stage one. Our method can handle the stochastic problem regardless of the number of stages and provide an alternative approach for the overlong setting.

The remainder of the study is presented as follows. In Section 2, the MSSP model is developed. The dimensionality reduction approach based on DE is presented in Section 3. Specifically, Section 3.1 focuses on the reduction of the scenario tree while Section 3.2 implements the transformation of the MSSP model accordingly. In Section 4, the application in one section of Yangtze River, China, is displayed and analyzed. The last section presents conclusions and remarks about some directions for future research.

\section{Problem Definition and Mathematical Formulation}

2.1. Problem Statement. Consider a basin, where a government prepares a construction plan of cascade hydropower stations to control flood or drought, satisfy irrigation, and pursue profits from power generation. Thus, the location and capacity decision-making problem of cascade hydropower stations is studied here, in which the long-term stochastic operations should be taken into account. The integrated MSSP model is formulated to optimize the total performance which involves the construction costs in the planning stage, as well as operational costs, penalty costs, and profits of power generation in the subsequent multiple stages.

Six decisions of the model can be divided into two groups. The first group should be made in the planning stage, including (1) the final selection of hydropower stations from the candidates; (2) the capacity of each selected hydropower station. The decisions in the second group are in each operational stage. They are (3) water storage of each selected hydropower station in each stage; (4) loss flow of each selected hydropower station in each stage; (5) water discharge to downstream from each hydropower station in each stage; and (6) water discharge of power generation in each selected hydropower station each stage. During these operational stages, we consider uncertain water inflows (including precipitation and inflows from tributaries) and water demands (from residents, agriculture, and industry). The number of stages, defined as $T$, could be extremely large.

As shown in Figure 1, given the locations of cascade hydropower stations, three scheduling activities: water discharge of power generation, abandoned water spill (go downstream directly without passing generator units) and loss flow should be periodically made. The water storage of each hydropower station is determined by its scheduling activities and random water inflows. Notice that the loss flow mentioned is discharged to local areas, rather than downstream. When the loss flow is larger than the actual demand, it would incur corresponding penalties. Moreover, the physical limits on the levels of water discharge, loss flow, and power generation of each hydropower station should be satisfied in each operational stage. 
TABLe 1: Model variables.

\begin{tabular}{|c|c|c|}
\hline Types of variables & Symbol & Description \\
\hline Sets & $R$ & Set of hydropower station candidates \\
\hline \multirow{15}{*}{ Deterministic variables } & $T$ & $\begin{array}{c}\text { The planning horizon is composed of } T \text { operational } \\
\text { stages }\end{array}$ \\
\hline & $R_{\min }$ & $\begin{array}{c}\text { The minimum required number of hydropower } \\
\text { stations in the plan }\end{array}$ \\
\hline & $e$ & Unit construction fee $\left(\mathrm{RMB} / \mathrm{m}^{3}\right)$ \\
\hline & $f$ & Unit profit of power generation $(\mathrm{RMB} / \mathrm{KWh})$ \\
\hline & $\Delta t$ & $\begin{array}{c}\text { Time interval of each operational stage (h, which is } \\
\text { the abbreviation of hour) }\end{array}$ \\
\hline & $c_{i}$ & $\begin{array}{c}\text { Penalty coefficient per unit in hydropower station } \\
\text { candidate } i\left(\mathrm{RMB} / \mathrm{m}^{3}\right)\end{array}$ \\
\hline & $K_{i}$ & Coefficient of output of candidate $i\left(\mathrm{~kg} /\left(\mathrm{m}^{2} \cdot \mathrm{s}^{2}\right)\right)$ \\
\hline & $N_{i \min }$ & The minimum output of candidate $i(\mathrm{~kW})$ \\
\hline & $N_{i \max }$ & The maximum output of candidate $i(\mathrm{~kW})$ \\
\hline & $H_{i}$ & Water head of candidate $i(\mathrm{~m})$ \\
\hline & $V_{i \min }$ & $\begin{array}{l}\text { The minimum required water storage of candidate } i \\
\text { when it is selected }\left(\mathrm{m}^{3}\right)\end{array}$ \\
\hline & $Q_{i \max }$ & $\begin{array}{c}\text { The maximum discharge limit of local area around } \\
\text { candidate } i\left(\mathrm{~m}^{3} / \mathrm{h}\right)\end{array}$ \\
\hline & $Q F_{\text {imax }}$ & The upper limit water discharge of candidate $i\left(\mathrm{~m}^{3} / \mathrm{h}\right)$ \\
\hline & $Q F_{i \min }$ & The lower limit water discharge of candidate $i\left(\mathrm{~m}^{3} / \mathrm{h}\right)$ \\
\hline & $r_{i}(t)$ & $\begin{array}{l}\text { Unit operational cost of candidate } i \text { at stage } t \text { (RMB/ } \\
\left.\left(\mathrm{m}^{3} \cdot \mathrm{h}\right)\right)\end{array}$ \\
\hline \multirow{4}{*}{ Stochastic variables } & $p^{s}$ & The probability of stagewise scenario $s$ \\
\hline & $\varepsilon_{i}^{s}(t)$ & $\begin{array}{l}\text { Outside water inflows in candidate } i \text { at stage } t \text { under } \\
\text { scenario } s\left(\mathrm{~m}^{3} / \mathrm{h}\right)\end{array}$ \\
\hline & $Q_{i \min }^{s}(t)$ & $\begin{array}{l}\text { Water demands in candidate } i \text { at stage } t \text { under } \\
\text { scenario } s\left(\mathrm{~m}^{3} / \mathrm{h}\right)\end{array}$ \\
\hline & $Q_{i \min }^{\prime s}(t)$ & $\begin{array}{l}\text { Acceptance level of water discharge in local area } \\
\text { surrounding candidate } i \text { at stage } t \text { under scenario } s \\
\qquad\left(\mathrm{~m}^{3} / \mathrm{h}\right)\end{array}$ \\
\hline
\end{tabular}

TABle 2: Decision variables.

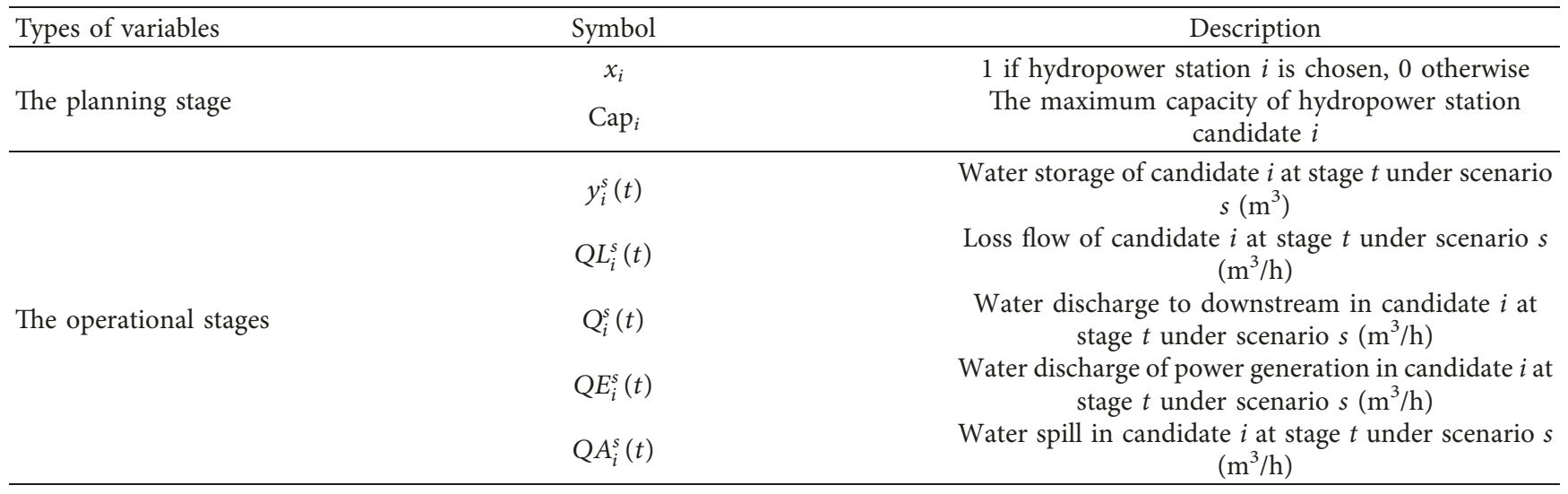

2.2. Model Formulation. Sets, exogenous deterministic and stochastic variables are given in Table 1 . Table 2 displays the decision variables in all stages.
The MSSP model which integrates the planning decisions and stochastic operational impacts is proposed as follows: 


$$
\begin{aligned}
\min & \sum_{i \in R} e \cdot \operatorname{Cap}_{i}+\sum_{t=1}^{T}\left(\Delta t \cdot \sum_{i \in R} r_{i}(t) \cdot \operatorname{Cap}_{i}\right) \\
& +\sum_{t=1}^{T} \sum_{s \in S}\left[p^{s} \cdot \sum_{i \in R}\left[\Delta t \cdot\left(c_{i} \cdot x_{i} \cdot\left(Q L_{i}^{s}(t)-Q_{i \min }^{\prime s}(t)\right)-f \cdot K_{i} \cdot Q E_{i}^{s}(t) \cdot H_{i}\right)\right]\right],
\end{aligned}
$$

s.t.

$$
\begin{aligned}
& R_{\min } \leq \sum_{i \in R} x_{i} \leq R, \\
& \mathrm{Cap}_{i} \leq M M \cdot x_{i}, \quad i \in R, \\
& Q E_{i}^{s}(t) \leq M M \cdot x_{i}, \quad t=1,2, \ldots, T ; i \in R ; s \in S, \\
& x_{i} \cdot N_{i \min } \leq K_{i} \cdot Q E_{i}^{s}(t) \cdot H_{i} \leq N_{i \max }, \quad t=1,2, \ldots, T ; i \in R ; s \in S, \\
& V_{i \min } \cdot x_{i} \leq y_{i}^{s}(t) \leq \mathrm{Cap}_{i}, \quad t=1,2, \ldots, T ; i \in R ; s \in S, \\
& y_{i}^{s}(t)-y_{i}^{s}(t-1)=\Delta t \cdot\left[Q_{i-1}^{s}(t)+\varepsilon_{i}^{s}(t)-Q_{i}^{s}(t)-Q L_{i}^{s}(t)\right], \quad t=1,2, \ldots, T ; i \in R ; s \in S, \\
& Q_{i}^{s}(t)=Q E_{i}^{s}(t)+Q A_{i}^{s}(t), \quad t=1,2, \ldots, T ; i \in R ; s \in S, \\
& Q F_{i \min } \leq Q E_{i}^{s}(t)+Q A_{i}^{s}(t) \leq Q F_{i \max }, \quad t=1,2, \ldots, T ; i \in R ; s \in S, \\
& Q_{i \min }^{s}(t) \leq Q L_{i}^{s}(t) \leq x_{i} \cdot Q_{i \max }+\left(1-x_{i}\right) \cdot Q_{i \min }^{s}(t), \quad t=1,2, \ldots, T ; i \in R ; s \in S, \\
& \operatorname{Cap}_{i}, y_{i}^{s}(t), Q L_{i}^{s}(t), Q E_{i}^{s}(t), Q Q_{i}^{s}(t), Q A_{i}^{s}(t) \geq 0, x_{i} \in\{0,1\}, \quad t=1,2, \ldots, T ; i \in R ; s \in S .
\end{aligned}
$$

The objective function (1a) is composed of the construction costs of all selected hydropower stations and the expected value of the operational costs, the environmental penalty costs, and the benefits of power generation under all the stagewise scenarios.

Constraint (1b) limits the number of hydropower stations to be chosen. Thus, Constraints (1c) and (1d) ensure that the corresponding maximum capacity and water discharge of unselected hydropower stations are zero in which $M M$ is a number big enough. Constraint (1e) gives the power generation limits. Constraint (1f) ensures that the water storage of the selected hydropower station is between the minimum required water storage and maximum capacity. Constraint (1g) gives water balance equations. Constraint (1h) shows the relationship between water discharge to downstream and water discharge of power generation, as well as water spill in each hydropower station candidate. Constraint (1i) presents the upper and lower limits of water discharge between two adjacent selected hydropower stations. Constraint $(1 \mathrm{j})$ guarantees that if the candidate is chosen, its loss flow should meet the local demand and cannot exceed the maximum discharge limit. And if a candidate is not selected, its loss flow is equal to the water demands. Constraint (1k) specifies the domains of the decision variables.

\section{Model Transformation}

Even the deterministic version of the proposed MSSP model is NP-hard. Besides, the dimension of stochastic scenarios in the MSSP model increases with the number of stages exponentially. It would further increase the solving difficulty. DE is a useful approach to handle the dimension problem of the large-scale setting [44]. It was first proposed in [42] to simplify the multistage deterministic convex optimization problem and then was applied to a multistage stochastic production-inventory programming model in [43]. The essence of DE is going to add up all the influence after a time point in the future by a so-called discount factor to compress stages. Hence, it coincides with our idea of aggregating the operational impacts on the planning decisions.

Next, we proposed a DE-based dimensionality reduction approach to address the above MSSP model. Specifically, because the size of the scenario tree is one of the main causes resulting in the computational difficulty, we first show how to compress the stages of the scenario tree at first (see Section 3.1). Along with the simplification of the scenario tree, we further show how to aggregate the operational decision variables and the deterministic parameters. With the compression of all these variables, the proposed MSSP 
model would be integrated into a two-stage one which is tractable (see Section 3.2).

3.1. Scenarios Generation and Simplification. Because the acceptance level of water discharge includes the quantity of water demands, we assume these two factors have a deterministic linear relationship. Thus, we focus on random water inflows and water demands here. Before we give the dimensionality reduction approach, we first show the generation and simplification procedures of stochastic scenarios by taking uncertain water inflows as an example. Uncertain water demands can be handled similarly.

We match each operational stage with a season. At seasonal stage $t=1,2, \ldots, T$, assume that $\xi=1,2$, and 3 correspond to three levels of random inflows: high, medium, and low, with the corresponding probability of $p_{1 H}, p_{1 M}$, and $p_{1 L}$, respectively. The quantities of water inflows in hydropower station candidate $i$ at stage $t$ with level $\xi$ can be represented as $\varepsilon_{i}^{\xi}(t), i \in R$. There are multiple stages considered in this study. Hence, each stagewise scenario of inflows, defined as $s_{1}\left(\in S_{1}\right)$, can be represented as a branch from $t=0 \sim T$ of the scenario tree, in which $t=0$ represents the planning stage. The corresponding probability $p\left(s_{1}\right)$ is the product of occurrence probabilities of all the nodes on this branch. Figure 2 gives the scenario tree faced by hydropower station candidate 1 .

As can be seen, the number of inflow scenarios of one candidate is up to $3^{T}$ in $T$ seasonal stages. For example, if we consider a 10 year horizon, the scenario size of inflows of one candidate would be $3^{40}\left(\approx 1.049 \times 10^{19}\right)$. Obviously, a simplification way is required. Here, we can remain the scenarios in early stages, while inflows in subsequent stages are replaced by corresponding constant estimated values. We do this for two reasons. The first is that the stochastic scenarios in subsequent stages are harder to give than these of early stages due to forecasting difficulty. Second, random inflows have intrinsic periodicity which means inflows in subsequent stages can be offset. Hence, an approximated way is to use the corresponding estimated values to replace the seasonal inflows in subsequent stages.

Thus, the scenario tree in Figure 2 is simplified to that in Figure 3 in which the uncertainties of $t=1 \sim 4$ remain. Denote the stagewise scenario in Figure 3 as $\widehat{s}_{1}$ and the corresponding scenario set as $\widehat{S}_{1}$. After simplification, the number of the decision variables is still large because of the big $T$. Hence, the dimensionality reduction approach is proposed to address this issue.

\subsubsection{Step 1 of Dimensionality Reduction-Stages Reduction} by Aggregating the Random Factors of the Same Season. The dimensionality reduction approach includes two steps. We focus on the first one in this section.

Due to the seasonal periodicity of inflows, we propose to aggregate the inflows of the same season together (see
Figure 4). Specifically, take the inflows of candidate 1 in the first stage $(t=1)$, i.e., $\varepsilon_{1}^{\xi}(1)$, as an example. Without loss of generality, assume that $T$ is the multiple of four. The subsequent water inflows with the same season, i.e., stage $4 \tau+1(\tau=1,2, \ldots,(T / 4)-1)$, have the same value, denoted as $\bar{\varepsilon}_{1}(1)$. Similarly, $\bar{\varepsilon}_{1}(2), \bar{\varepsilon}_{1}(3)$, and $\bar{\varepsilon}_{1}(4)$ represent other three seasonal values of water inflows at candidate 1 . Referring to the concept of present value, we use factor $\delta$ $(0<\delta<1)$ to discount the impacts of subsequent stages. Thus, the corresponding aggregated water inflows of candidate 1 in the first stage $(t=1)$ under the case of $\xi$, denoted as $\varepsilon_{1}^{* \xi}(1)$, are the discounted value which can be expressed as

$$
\begin{aligned}
\varepsilon_{1}^{* \xi}(1) & =\varepsilon_{1}^{\xi}(1)+\delta^{4} \cdot \bar{\varepsilon}_{1}(1)+\cdots+\delta^{4 \tau} \cdot \bar{\varepsilon}_{1}(1)+\cdots+\delta^{T-4} \cdot \bar{\varepsilon}_{1}(1) \\
& =\varepsilon_{1}^{\xi}(1)+\bar{\varepsilon}_{1}(1) \cdot \frac{1-\delta^{T}}{1-\delta^{4}}, \quad \tau=1,2, \ldots, \frac{T}{4}-1 .
\end{aligned}
$$

Then, $\varepsilon_{1}^{* \xi}(2), \varepsilon_{1}^{* \xi}(3)$, and $\varepsilon_{1}^{* \xi}(4)$ can be obtained similarly. Thus, the scenario tree in Figure 3 is compressed into Figure 5, in which the value associated with each node is a discounted value now. However, the number of branches $\left(\widehat{s}_{1}\right)$ and their corresponding occurrence probabilities $\left(p\left(\widehat{s}_{1}\right)\right)$ remain unchanged.

3.1.2. Step 2 of Dimensionality Reduction-Stages Reduction Across Seasons. It is worth recalling that this study focuses on the planning problem with the consideration of future operational activities under uncertainties. Thus, the second step of the dimensionality reduction approach is to implement the further aggregation of the impacts of operational stages together. In other words, the random variables $(t=2,3,4)$ on a branch in Figure 5 will be integrated into the first operational stage $(t=1)$. For the example that the inflows of candidate 1 under stagewise scenario $\widehat{s}_{1}$ which is composed of four high-level stages (i.e., $\xi=1$ ), its present value can be given as

$$
\varepsilon_{1}^{* \widehat{s}_{1}}=\varepsilon_{1}^{* 1}(1)+\delta \cdot \varepsilon_{1}^{* 1}(2)+\delta^{2} \cdot \varepsilon_{1}^{* 1}(3)+\delta^{3} \cdot \varepsilon_{1}^{* 1}(4) .
$$

Although the impacts of scenario $\widehat{s}_{1}$ is aggregated now, such process would not change the number of scenario $\widehat{s}_{1}$ and its probability $p\left(\widehat{s}_{1}\right)$.

The stochastic scenario of random water demands could be handled similarly, and the corresponding final scenario set is denoted as $\widehat{S}_{2}$ which is composed by scenario $\widehat{s}_{2}$ with the probability $p\left(\widehat{s}_{2}\right)$. Hence, the set of total scenarios $S$ used in the proposed MSSP model is simplified to $\widehat{S}$, which is the Cartesian product of $\widehat{S}_{1}$ and $\widehat{S}_{2}$ :

$$
\widehat{S}=\widehat{S}_{1} \times \widehat{S}_{2}=\left\{\left(\widehat{s}_{1}, \widehat{s}_{2}\right) \mid \widehat{s}_{1} \in \widehat{S}_{1}, \widehat{s}_{2} \in \widehat{S}_{2}\right\} .
$$

The corresponding probability of new scenario $\widehat{\mathcal{S}}(\in \widehat{S})$, which is the union of $\widehat{s}_{1}$ and $\widehat{s}_{2}$, can be calculated as

$$
p(\widehat{s})=p\left(\widehat{s}_{1}\right) \times p\left(\widehat{s}_{2}\right) .
$$




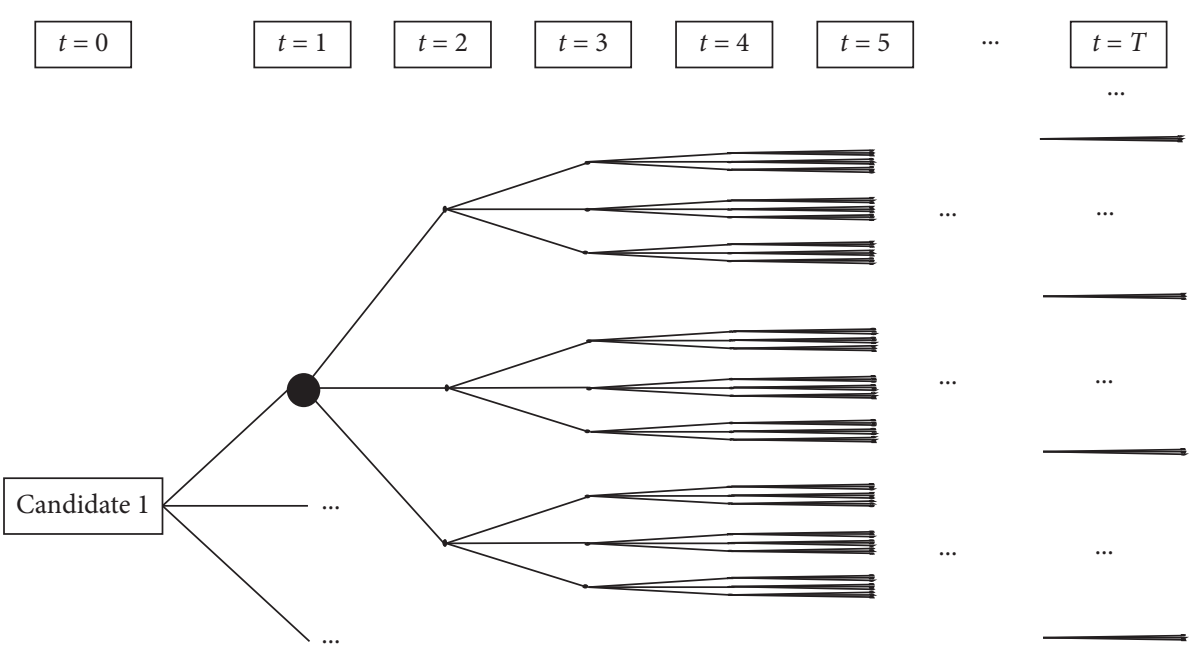

Figure 2: Original multistage scenario tree (random inflows of candidate 1).

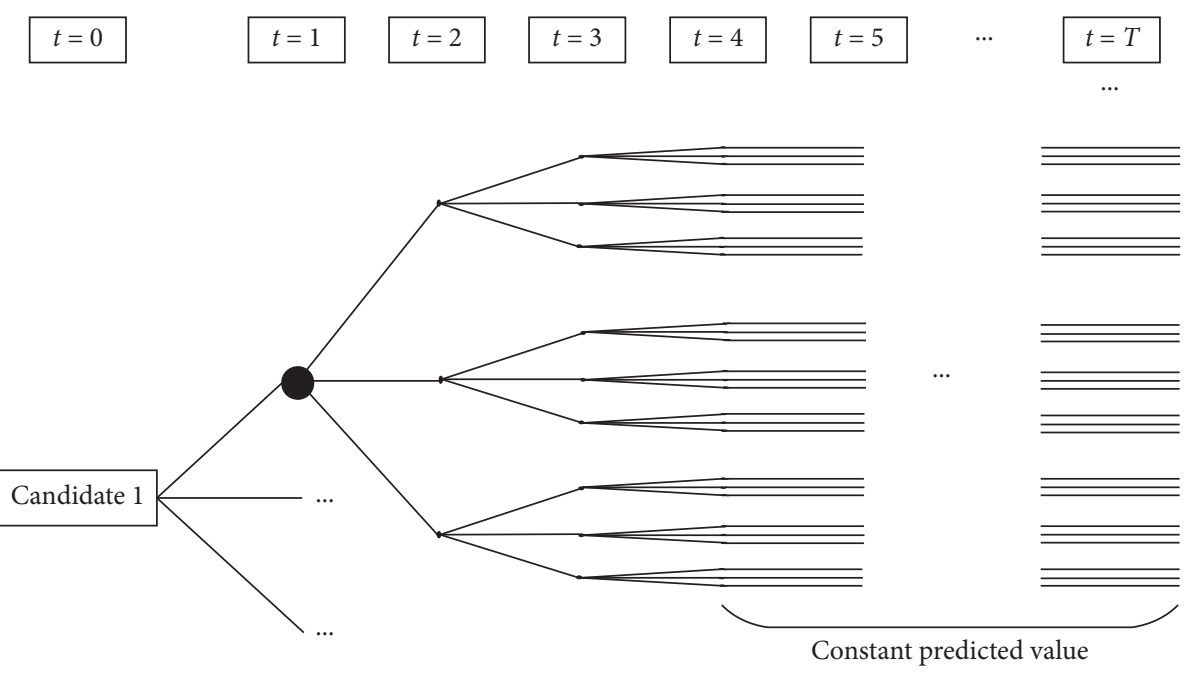

FIGURE 3: Simplified multistage scenario tree (random inflows of candidate 1).

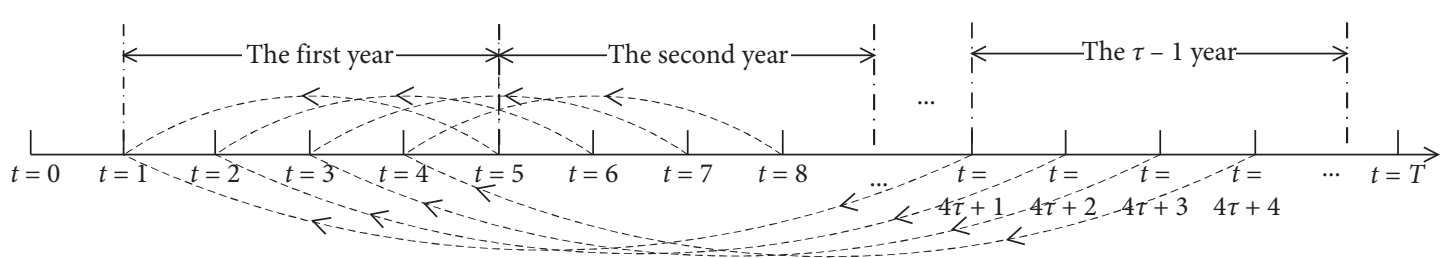

FIGURE 4: Illustration of stage reduction by aggregating the random factors of the same season.

Till now, the stagewise scenario tree is compressed into a one-stage scenario set $\widehat{S}$.

3.2. Transformation of the MSSP Model. The above work simplifies the scenario tree by discounting the random variables. Accordingly, with the compression of the scenario tree, the deterministic parameters and the decision variables should also be aggregated, and thus, the MSSP model would be simplified. The transformation process also includes two steps. 


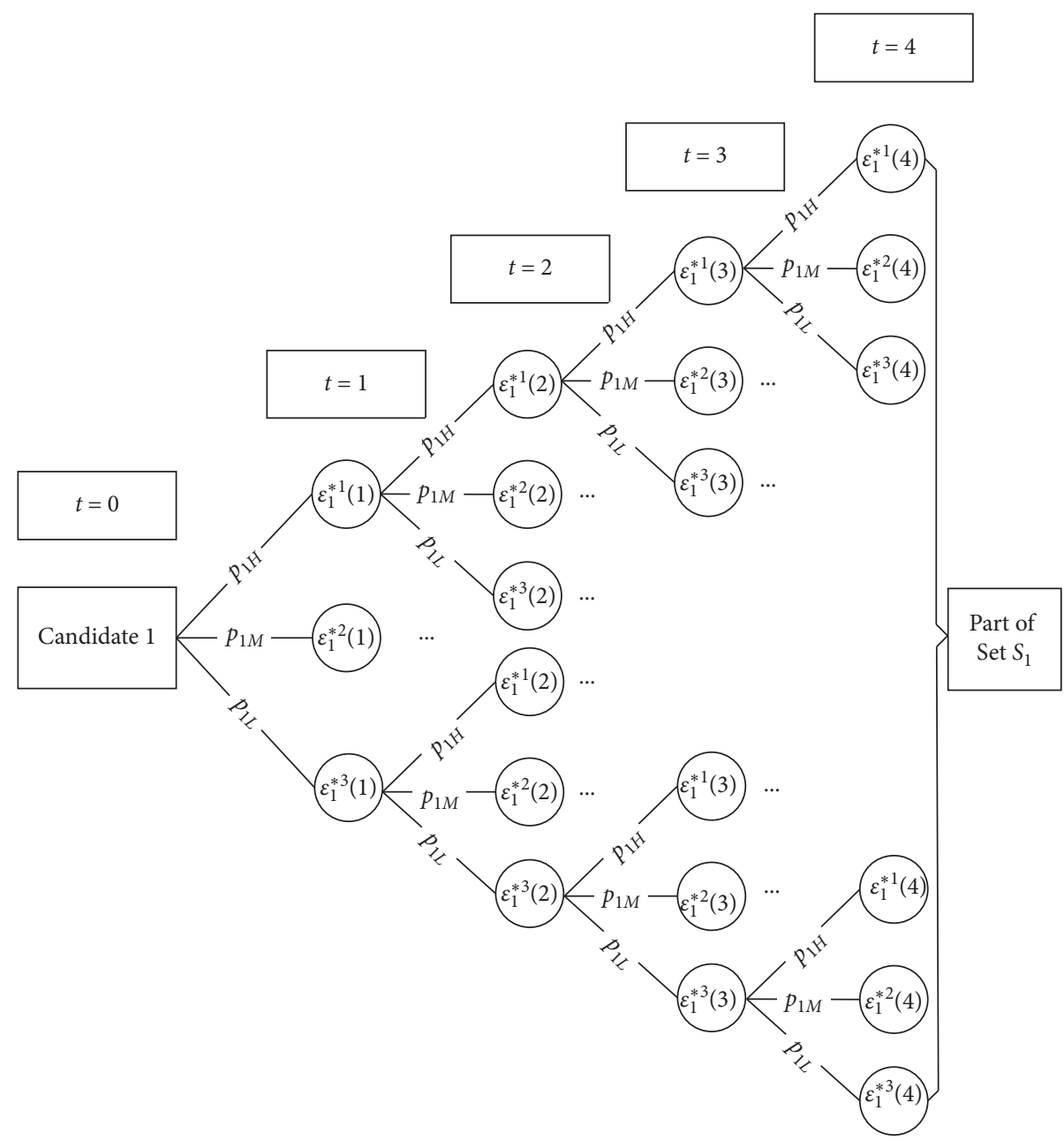

FIgURE 5: Scenario tree (random inflows of candidate 1).

3.2.1. Step 1 of Dimensionality Reduction. At first, we decompose the operational stages of MSSP model into multiple submodels according to each stagewise scenario $s$ $(\in S)$. Then, such submodel is not only multistage but also deterministic because it is given based on the independent scenario. For the submodel, we show how to simplify the variables and the model along with the reduction of the stages.

To facilitate the implementation of DE, we first reformulate the submodel as the following equivalent:

$\min \sum_{j=1}^{4} \sum_{\tau=0}^{(T / 4)-1}\left\{\sum_{i \in R} \Delta t \cdot\left[c_{i} \cdot x_{i} \cdot\left(Q L_{i}(4 \tau+j)-Q_{i \min }^{\prime}(4 \tau+j)\right)-f \cdot K_{i} \cdot Q E_{i}(4 \tau+j) \cdot H_{i}\right]\right\}$,

s.t.

$$
\begin{aligned}
& Q E_{i}(4 \tau+j) \leq M M \cdot x_{i}, \quad i \in R ; \tau=0, \ldots, \frac{T}{4}-1 ; j=1,2,3,4, \\
& x_{i} \cdot N_{i \min } \leq K_{i} \cdot Q E_{i}(4 \tau+j) \cdot H_{i} \leq N_{i \max }, \quad i \in R ; \tau=0, \ldots, \frac{T}{4}-1 ; j=1,2,3,4, \\
& V_{i \min } \cdot x_{i} \leq y_{i}(4 \tau+j) \leq \mathrm{Cap}_{i}, \quad i \in R ; \tau=0, \ldots, \frac{T}{4}-1 ; j=1,2,3,4,
\end{aligned}
$$




$$
\begin{aligned}
& y_{i}(4 \tau+j)-y_{i}(4 \tau+j-1)=\Delta t \cdot\left[Q_{i-1}(4 \tau+j)+\varepsilon_{i}(4 \tau+j)-Q_{i}(4 \tau+j)-Q L_{i}(4 \tau+j)\right] \text {, } \\
& i \in R ; \tau=0, \ldots, \frac{T}{4}-1 ; j=1,2,3,4 \text {, } \\
& Q_{i}(4 \tau+j)=Q E_{i}(4 \tau+j)+Q A_{i}(4 \tau+j), \quad i \in R ; \tau=0, \ldots, \frac{T}{4}-1 ; j=1,2,3,4, \\
& Q F_{i \min } \leq Q E_{i}(4 \tau+j)+Q A_{i}(4 \tau+j) \leq Q F_{i \max }, \quad i \in R ; \tau=0, \ldots, \frac{T}{4}-1 ; j=1,2,3,4, \\
& Q_{i \min }(4 \tau+j) \leq Q L_{i}(4 \tau+j) \leq x_{i} \cdot Q_{i \max }+\left(1-x_{i}\right) \cdot Q_{i \min }(4 \tau+j), \\
& i \in R ; \tau=0, \ldots, \frac{T}{4}-1 ; j=1,2,3,4 \text {. }
\end{aligned}
$$

Step 1. Here, we focus on the objective. Specifically, similar to formula (2), the objective values at stage $4 \tau+j$ $(\tau=0,1, \ldots,(T / 4)-1, j=1,2,3,4)$ also should be compressed into the corresponding stage $j$. Thus, with the introduction of the discount factor $\delta$, (6a) can be reformulated as (7):

$$
\min \sum_{j=1}^{4} \sum_{\tau=0}^{(T / 4)-1}\left\{\delta^{4 \tau} \cdot \sum_{i \in R} \Delta t \cdot\left[c_{i} \cdot x_{i} \cdot\left(Q L_{i}(4 \tau+j)-Q_{i \min }^{\prime}(4 \tau+j)\right)-f \cdot K_{i} \cdot Q E_{i}(4 \tau+j) \cdot H_{i}\right]\right\}
$$

Step 2. Along with simplification of the scenario tree, the decision variables and the deterministic parameters should also be aggregated. Because they exist in both the objective and the constraints, to facilitate our transformation, we integrate the constraints into the objective. Specifically, we use dual multipliers $\beta_{k, 4 \tau+j}(k=1, \ldots, 7)$ to relax the $k$-th one of Constraints (6b) (6h) of stage $4 \tau+j(\tau=0,1, \ldots,(T / 4)-1$, $j=1,2,3,4)$. Then, the optimization problem $((6 \mathrm{~b}) \sim(6 \mathrm{~h})$, (7)) can be transformed into the unconstrainted problem (8):

$$
\begin{aligned}
\min & \sum_{j=1}^{4} \sum_{\tau=0}^{(T / 4)-1}\left\{\delta^{4 \tau} \cdot \sum_{i \in R} \Delta t \cdot\left[c_{i} \cdot x_{i} \cdot\left(Q L_{i}(4 \tau+j)-Q_{i \min }^{\prime}(4 \tau+j)\right)-f \cdot K_{i} \cdot Q E_{i}(4 \tau+j) \cdot H_{i}\right]\right. \\
& +\beta_{1,4 \tau+j}^{+} \cdot\left(Q E_{i}(4 \tau+j)-M M \cdot x_{i}\right)+\beta_{2,4 \tau+j}^{+} \cdot\left(K_{i} \cdot Q E_{i}(4 \tau+j) \cdot H_{i}-N_{i \max }\right)+\beta_{2,4 \tau+j}^{-} \\
& \cdot\left(K_{i} \cdot Q E_{i}(4 \tau+j) \cdot H_{i}-x_{i} \cdot N_{i \min }\right)+\beta_{3,4 \tau+j}^{+} \cdot\left(y_{i}(4 \tau+j)-C a p_{i}\right)+\beta_{3,4 \tau+j}^{-}(j) \cdot\left(y_{i}(4 \tau+j)-V_{i \min } \cdot x_{i}\right) \\
& +\beta_{4,4 \tau+j} \cdot\left(y_{i}(4 \tau+j)-y_{i}(4 \tau+j-1)-\Delta t \cdot\left(Q_{i-1}(4 \tau+j)+\varepsilon_{i}(4 \tau+j)-Q_{i}(4 \tau+j)-Q L_{i}(4 \tau+j)\right)\right) \\
& +\beta_{5,4 \tau+j} \cdot\left(Q_{i}(4 \tau+j)-Q E_{i}(4 \tau+j)-Q A_{i}(4 \tau+j)\right)+\beta_{6,4 \tau+j}^{+} \cdot\left(Q E_{i}(4 \tau+j)+Q A_{i}(4 \tau+j)-Q F_{i \max }\right) \\
& +\beta_{6,4 \tau+j}^{-} \cdot\left(Q E_{i}(4 \tau+j)+Q A_{i}(4 \tau+j)-Q F_{i \min }\right)+\beta_{7,4 \tau+j}^{+} \cdot\left(Q L_{i}(4 \tau+j)-x_{i} \cdot Q Q_{i \max }-\left(1-x_{i}\right) \cdot Q_{i \min }(4 \tau+j)\right) \\
& \left.+\beta_{7,4 \tau+j}^{-} \cdot\left(Q L_{i}(4 \tau+j)-Q Q_{i \min }(4 \tau+j)\right)\right\}
\end{aligned}
$$

The DE approach assumes the dual multipliers have the linear relationship with the discount factor $\delta$ [42]. Thus, let the dual multipliers here take the form $\beta_{k, 4 \tau+j}=\delta^{4 \tau} \cdot \beta_{k}(j)$. Then, problem (8) can further be reformulated as 


$$
\begin{aligned}
& \min \sum_{j=1}^{4} \sum_{\tau=0}^{(T / 4)-1} \delta^{4 \tau} \cdot\left\{\sum_{i \in R} \Delta t \cdot\left[c_{i} \cdot x_{i} \cdot\left(Q L_{i}(4 \tau+j)-Q_{i \min }^{\prime}(4 \tau+j)\right)-f \cdot K_{i} \cdot Q E_{i}(4 \tau+j) \cdot H_{i}\right]+\beta_{1}^{+}(j) \cdot\left(Q E_{i}(4 \tau+j)-M M \cdot x_{i}\right)\right. \\
& \quad+\beta_{2}^{+}(j) \cdot\left(K_{i} \cdot Q E_{i}(4 \tau+j) \cdot H_{i}-N_{i \max }\right)+\beta_{2}^{-}(j) \cdot\left(K_{i} \cdot Q E_{i}(4 \tau+j) \cdot H_{i}-x_{i} \cdot N_{i \min }\right)+\beta_{3}^{+}(j) \cdot\left(y_{i}(4 \tau+j)-C a p_{i}\right) \\
& +\beta_{3}^{-}(j) \cdot\left(y_{i}(4 \tau+j)-V_{i \min } \cdot x_{i}\right)+\beta_{4}(j) \cdot\left(y_{i}(4 \tau+j)-y_{i}(4 \tau+j-1)-\Delta t \cdot\left(Q_{i-1}(4 \tau+j)+\varepsilon_{i}(4 \tau+j)\right.\right. \\
& \left.\left.\quad-Q_{i}(4 \tau+j)-Q L_{i}(4 \tau+j)\right)\right)+\beta_{5}(j) \cdot\left(Q_{i}(4 \tau+j)-Q E_{i}(4 \tau+j)-Q A_{i}(4 \tau+j)\right)+\beta_{6}^{+}(j) \cdot\left(Q E_{i}(4 \tau+j)\right. \\
& \left.+Q A_{i}(4 \tau+j)-Q F_{i \max }\right)+\beta_{6}^{-}(j) \cdot\left(Q E_{i}(4 \tau+j)+Q A_{i}(4 \tau+j)-Q F_{i \min }\right) \\
& \left.+\beta_{7}^{+}(j) \cdot\left(Q L_{i}(4 \tau+j)-x_{i} \cdot Q_{i \max }-\left(1-x_{i}\right) \cdot Q_{i \min }(4 \tau+j)\right)+\beta_{7}^{-}(j) \cdot\left(Q L_{i}(4 \tau+j)-Q_{i \min }(4 \tau+j)\right)\right\} .
\end{aligned}
$$

Step 3. Thus, similar to the discounting way of the random variables in Section 3.1.1, we can integrate the decision variables and the parameters under the same season together by $\delta$. To make the above model concise, the so-called integrated' primal variables are introduced to replace the corresponding discounted values. For example,

$$
Q L_{i}^{*}(j)=\sum_{\tau=0}^{(T / 4)-1} \delta^{4 \tau} \cdot Q L_{i}(4 \tau+j), \quad j=1,2,3,4 ; i \in R,
$$

$$
Q_{i \max }^{*}=\sum_{\tau=0}^{(T / 4)-1} \delta^{4 \tau} \cdot Q_{i \max }, \quad i \in R .
$$

Other integrated primal variables can be given similarly, and all of them take an asterisk at their top right corner. Thus, with the introduction of the 'integrated' primal variables, the symbol $\sum_{\tau=0}^{(T / 4)-1}(\cdot)$ in Objective (9) could be removed, and then, the operational stages $5 \sim T$ in the submodel could be compressed into the stages $1 \sim 4$, respectively. Thus, we have

$$
\begin{aligned}
\min & \sum_{j=1}^{4}\left\{\sum_{i \in R} \Delta t \cdot\left[c_{i} \cdot x_{i} \cdot\left(Q L_{i}^{*}(j)-Q_{i \min }^{\prime *}(j)\right)-f \cdot K_{i} \cdot Q E_{i}^{*}(j) \cdot H_{i}\right]\right. \\
& +\beta_{1}^{+}(j) \cdot\left(Q E_{i}^{*}(j)-M M \cdot x_{i}\right)+\beta_{2}^{+}(j) \cdot\left(K_{i} \cdot Q E_{i}^{*}(j) \cdot H_{i}-N_{i \max }^{*}\right)+\beta_{2}^{-}(j) \cdot\left(K_{i} \cdot Q E_{i}^{*}(j) \cdot H_{i}-x_{i} \cdot N_{i \min }^{*}\right) \\
& +\beta_{3}^{+}(j) \cdot\left(y_{i}^{*}(j)-C a p_{i}^{*}\right)+\beta_{3}^{-}(j) \cdot\left(y_{i}^{*}(j)-V_{i \min }^{*} \cdot x_{i}\right) \\
& +\beta_{4}(j) \cdot\left(y_{i}^{*}(j)-y_{i}^{*}(j-1)-\Delta t \cdot\left(Q_{i-1}^{*}(j)+\varepsilon_{i}^{*}(j)-Q_{i}^{*}(j)-Q L_{i}^{*}(j)\right)\right)+\beta_{5}(j) \cdot\left(Q_{i}^{*}(j)-Q E_{i}^{*}(j)-Q A_{i}^{*}(j)\right) \\
& +\beta_{6}^{+}(j) \cdot\left(Q E_{i}^{*}(j)+Q A_{i}^{*}(j)-Q F_{i \max }^{*}\right)+\beta_{6}^{-}(j) \cdot\left(Q E_{i}^{*}(j)+Q A_{i}^{*}(j)-Q F_{i \min }^{*}\right) \\
& \left.+\beta_{7}^{+}(j) \cdot\left(Q L_{i}^{*}(j)-x_{i} \cdot Q_{i \max }^{*}-\left(1-x_{i}\right) \cdot Q_{i \min }^{*}(j)\right)+\beta_{7}^{-}(j) \cdot\left(Q L_{i}^{*}(j)-Q_{i \min }^{*}(j)\right)\right\} .
\end{aligned}
$$

Step 4. Notice that the above transformation is for the submodel which is under a specific scenario. Specifically, Step 2 relaxes the constraints to get a unconstrainted model, and then, Step 3 aggregates the multistage for this model.
Here, we need to extract the relaxed constraints back at first and then combine the model with each scenario $\widehat{s}(\in \widehat{S})$ (see Figure 5) and the planning stage. Thus, we can get the following intermediate model: 


$$
\begin{aligned}
\min & \sum_{i \in R} e \cdot \operatorname{Cap}_{i}+\sum_{j=1}^{4}\left(\Delta t \cdot \sum_{i \in R} r_{i}^{*}(j) \cdot \operatorname{Cap}_{i}\right) \\
& +\sum_{\widehat{s} \in \widehat{S}} p^{\widehat{s}} \cdot \sum_{j=1}^{4} \sum_{i \in R} \Delta t \cdot\left[c_{i} \cdot x_{i} \cdot\left(Q L_{i}^{* \widehat{s}}(j)-Q_{i \min }^{* \widehat{s}}(j)\right)-f \cdot K_{i} \cdot Q E_{i}^{* \widehat{s}}(j) \cdot H_{i}\right],
\end{aligned}
$$

s.t.

$$
\begin{aligned}
& R_{\min } \leq \sum_{i \in R} x_{i} \leq R, \\
& \operatorname{Cap}_{i} \leq M M \cdot x_{i}, \quad i \in R, \\
& Q E_{i}^{* \widehat{s}}(j) \leq M M \cdot x_{i}, \quad i \in R ; \widehat{s} \in \widehat{S} ; j=1,2,3,4, \\
& x_{i} \cdot N_{i \min }^{*} \leq K_{i} \cdot Q E_{i}^{* \widehat{s}}(j) \cdot H_{i} \leq N_{i \max }^{*}, \quad i \in R ; \widehat{s} \in \widehat{S} ; j=1,2,3,4, \\
& V_{i \min }^{*} \cdot x_{i} \leq y_{i}^{* \widehat{s}}(j) \leq \operatorname{Cap}_{i}^{*}, \quad i \in R ; \widehat{s} \in \widehat{S} ; j=1,2,3,4, \\
& y_{i}^{* \widehat{s}}(j)-y_{i}^{* \widehat{s}}(j-1)=\Delta t \cdot\left[Q_{i-1}^{* \widehat{s}}(j)+\varepsilon_{i}^{* \widehat{s}}(j)-Q_{i}^{* \widehat{s}}(j)-Q L_{i}^{* \widehat{s}}(j)\right], \\
& Q_{i}^{* \widehat{s}}(j)=Q E_{i}^{* \widehat{s}}(j)+Q A_{i}^{* \widehat{s}}(j), \quad i \in R ; \widehat{s} \in \widehat{S} ; j=1,2,3,4, \widehat{s} \in \hat{S} ; j=1,2,3,4, \\
& Q F_{i \min }^{*} \leq Q E_{i}^{* \widehat{s}}(j)+Q A_{i}^{* s}(j) \leq Q F_{i \max }^{*}, i \in R ; \widehat{s} \in \widehat{S} ; j=1,2,3,4, \\
& Q_{i \min }^{* \widehat{s}}(j) \leq Q L_{i}^{* \widehat{s}}(j) \leq x_{i} \cdot Q_{i \max }^{*}+\left(1-x_{i}\right) \cdot Q_{i \min }^{* \widehat{s}}(j), \quad i \in R ; \widehat{s} \in \widehat{S} ; j=1,2,3,4, \\
& x_{i} \in\{0,1\}, \operatorname{Cap}_{i} \geq 0, \quad i \in R,
\end{aligned}
$$

in which the primal variables $\mathrm{Cap}_{i}^{*}$ can be given as follows:

$$
\begin{aligned}
\operatorname{Cap}_{i}^{*} & =\sum_{\tau=0}^{(T / 4)-1} \delta^{4 \tau} \cdot \operatorname{Cap}_{i}=\operatorname{Cap}_{i} \cdot\left(\delta^{0}+\delta^{4}+\delta^{8}+\cdots+\delta^{T-8}+\delta^{T-4}\right) \\
& =\operatorname{Cap}_{i} \cdot \frac{1-\delta^{T}}{1-\delta^{4}} \approx \operatorname{Cap}_{i} \cdot \frac{1}{1-\delta^{4}} ; \quad i \in R,
\end{aligned}
$$

in which $\delta^{T}$ can be approximated as zero when the number of stages $T$ is large enough. Similarly, the parameters $r_{i}^{*}(j)$ could be given as

$$
r_{i}^{*}(j)=\sum_{\tau=0}^{(T / 4)-1} \delta^{4 \tau} \cdot r_{i}(4 \tau+j), \quad j=1,2,3,4 ; i \in R .
$$

We can assume the unit operational cost of candidate $i$ at each stage $t$ is the same, i.e., $r_{i}$. Then, $(12 \mathrm{~m})$ can be further represented as

$$
r_{i}^{*}(j)=r_{i} \cdot \frac{1-\delta^{T}}{1-\delta^{4}} \approx r_{i} \cdot \frac{1}{1-\delta^{4}}, \quad i \in R .
$$

Moreover, it is easy to know that $\operatorname{Cap}_{i}^{*}, y_{i}^{* \widehat{s}}(j), Q L_{i}^{* \widehat{s}}(j)$, $Q E_{i}^{* \widehat{s}}(j), Q_{i}^{* \widehat{s}}(j)$, an $\mathrm{d} Q A_{i}^{* \widehat{s}}(j)$ are all nonnegative.

Till now, along with the stage reduction as shown in Section 3.1.1, the T-stage operations of the MSSP model is integrated into a four-stage model. The corresponding scenario tree of this intermediate model is shown in Figure 5.

3.2.2. Step 2 of Dimensionality Reduction. Further model transformation is required due to the stage reduction in Section 3.1.2. Notice that $j(=1,2,3,4)$ in the intermediate model (12) refers to the first four operational stages. Here, we use symbol $t$ to replace the corresponding $j$ and then integrate stage $t=2,3,4$ into the first stage $(t=1)$ as illustrated in Section 3.1.2. The way how to transform is similar to that in Section 3.2.1.

We still focus on the operational stage under a specific stagewise scenario. Thus, the corresponding submodel can be described as follows: 


$$
\begin{array}{ll}
\min & \sum_{t=1}^{4} \sum_{i \in R}\left\{\Delta t \cdot\left[c_{i} \cdot x_{i} \cdot\left(Q L_{i}^{*}(t)-Q_{i \min }^{\prime *}(t)\right)-f \cdot K_{i} \cdot Q E_{i}^{*}(t) \cdot H_{i}\right]\right\}, \\
\text { s.t. } & \\
& Q E_{i}^{*}(t) \leq M M \cdot x_{i}, \quad i \in R ; t=1,2,3,4, \\
& x_{i} \cdot N_{i \min }^{*} \leq K_{i} \cdot Q E_{i}^{*}(t) \cdot H_{i} \leq N_{i \max }^{*}, \quad i \in R ; t=1,2,3,4, \\
& V_{i \min }^{*} \cdot x_{i} \leq y_{i}^{*}(t) \leq \frac{1}{1-\delta^{4}} \cdot \mathrm{Cap}_{i}, \quad i \in R ; t=1,2,3,4, \\
& y_{i}^{*}(t)-y_{i}^{*}(t-1)=\Delta t \cdot\left[Q_{i-1}^{*}(t)+\varepsilon_{i}^{*}(t)-Q_{i}^{*}(t)-Q L_{i}^{*}(t)\right], \\
& Q_{i}^{*}(t)=Q E_{i}^{*}(t)+Q A_{i}^{*}(t), \quad i \in R ; t=1,2,3,4, \\
& Q F_{i \min }^{*} \leq Q E_{i}^{*}(t)+Q A_{i}^{*}(t) \leq Q F_{i \max }^{*}, \quad i \in R ; t=1,2,3,4, \\
& Q_{i \min }^{*}(t) \leq Q L_{i}^{*}(t) \leq x_{i} \cdot Q_{i \max }^{*}+\left(1-x_{i}\right) \cdot Q_{i \min }^{*}(t), \quad i \in R ; t=1,2,3,4,
\end{array}
$$

Step 5. The discount factor $\delta$ is still used to aggregate the value of the objective function at stage $t(=2,3,4)$. Then, the objective function (13a) is transformed as

$$
\min \sum_{t=1}^{4}\left\{\delta^{t-1} \cdot \sum_{i \in R} \Delta t \cdot\left[c_{i} \cdot x_{i} \cdot\left(Q L_{i}(t)-Q_{i \min }^{\prime}(t)\right)-f \cdot K_{i} \cdot Q E_{i}(t) \cdot H_{i}\right]\right\} .
$$

Step 6. Similar to Step 2, use dual multipliers $\beta_{7+k, t}(k=1$, $\ldots, 7)$, to relax the $k$-th one of Constraints $(13 \mathrm{~b}) \sim(13 \mathrm{~h})$ of stage $t$. Thus, the operational optimization problem under stage $t=1 \sim 4((13 \mathrm{~b}) \sim(13 \mathrm{~h}),(14))$ can be transformed to an unconstrainted one:

$$
\begin{aligned}
\min & \sum_{t=1}^{4}\left\{\delta^{t-1} \cdot \sum_{i \in R} \Delta t \cdot\left[c_{i} \cdot x_{i} \cdot\left(Q L_{i}^{*}(t)-Q_{i \min }^{\prime *}(t)\right)-f \cdot K_{i} \cdot Q E_{i}^{*}(t) \cdot H_{i}\right]\right. \\
& +\beta_{8, t}^{+} \cdot\left(Q E_{i}^{*}(t)-M M \cdot x_{i}\right) \\
& +\beta_{9, t}^{+} \cdot\left(K_{i} \cdot Q E_{i}^{*}(t) \cdot H_{i}-N_{i \max }^{*}\right)+\beta_{9, t}^{-} \cdot\left(K_{i} \cdot Q E_{i}^{*}(t) \cdot H_{i}-x_{i} \cdot N_{i \min }^{*}\right) \\
& +\beta_{10, t}^{+} \cdot\left(y_{i}^{*}(t)-\frac{1}{1-\delta^{4}} \cdot C a p_{i}\right)+\beta_{10, t}^{-} \cdot\left(y_{i}^{*}(t)-V_{i \min }^{*} \cdot x_{i}\right) \\
& +\beta_{11, t} \cdot\left(y_{i}^{*}(t)-y_{i}^{*}(t-1)-\Delta t \cdot\left(Q_{i-1}^{*}(t)+\varepsilon_{i}^{*}(t)-Q_{i}^{*}(t)-Q L_{i}^{*}(t)\right)\right) \\
& +\beta_{12, t} \cdot\left(Q_{i}^{*}(t)-Q E_{i}^{*}(t)-Q A_{i}^{*}(t)\right) \\
& +\beta_{13, t}^{+} \cdot\left(Q E_{i}^{*}(t)+Q A_{i}^{*}(t)-Q F_{i \max }^{*}\right) \\
& +\beta_{13, t}^{-} \cdot\left(Q E_{i}^{*}(t)+Q A_{i}^{*}(t)-Q F_{i \min }^{*}\right) \\
& \left.+\beta_{14, t}^{+} \cdot\left(Q L_{i}^{*}(t)-x_{i} \cdot Q_{i \max }^{*}-\left(1-x_{i}\right) \cdot Q_{i \min }^{*}(t)\right)+\beta_{14, t}^{-} \cdot\left(Q L_{i}^{*}(t)-Q_{i \min }^{*}(t)\right)\right\}
\end{aligned}
$$


in which the dual multipliers follow the form $\beta_{7+k, t}=\delta^{t-1} \cdot \beta_{7+k}$. Hence, problem (15) can further be expressed as

$$
\begin{aligned}
\min & \sum_{t=1}^{4} \delta^{t-1} \cdot\left\{\sum_{i \in R} \Delta t \cdot\left[c_{i} \cdot x_{i} \cdot\left(Q L_{i}^{*}(t)-Q_{i \min }^{\prime *}(t)\right)-f \cdot K_{i} \cdot Q E_{i}^{*}(t) \cdot H_{i}\right]\right. \\
& +\beta_{8}^{+} \cdot\left(Q E_{i}^{*}(t)-M M \cdot x_{i}\right) \\
& +\beta_{9}^{+} \cdot\left(K_{i} \cdot Q E_{i}^{*}(t) \cdot H_{i}-N_{i \max }^{*}\right)+\beta_{9}^{-} \cdot\left(K_{i} \cdot Q E_{i}^{*}(t) \cdot H_{i}-x_{i} \cdot N_{i \min }^{*}\right) \\
& +\beta_{10}^{+} \cdot\left(y_{i}^{*}(t)-\frac{1}{1-\delta^{4}} \cdot \mathrm{Cap}_{i}\right)+\beta_{10}^{-} \cdot\left(y_{i}^{*}(t)-V_{i \min }^{*} \cdot x_{i}\right) \\
& +\beta_{11} \cdot\left(y_{i}^{*}(t)-y_{i}^{*}(t-1)-\Delta t \cdot\left(Q_{i-1}^{*}(t)+\varepsilon_{i}^{*}(t)-Q_{i}^{*}(t)-Q L_{i}^{*}(t)\right)\right) \\
& +\beta_{12} \cdot\left(Q_{i}^{*}(t)-Q E_{i}^{*}(t)-Q A_{i}^{*}(t)\right)+\beta_{13}^{+} \cdot\left(Q E_{i}^{*}(t)+Q A_{i}^{*}(t)-Q F_{i \max }^{*}\right) \\
& +\beta_{13}^{-} \cdot\left(Q E_{i}^{*}(t)+Q A_{i}^{*}(t)-Q F_{i \min }^{*}\right) \\
& \left.+\beta_{14}^{+} \cdot\left(Q L_{i}^{*}(t)-x_{i} \cdot Q_{i \max }^{*}-\left(1-x_{i}\right) \cdot Q_{i \min }^{*}(t)\right)+\beta_{14}^{-} \cdot\left(Q L_{i}^{*}(t)-Q_{i \min }^{*}(t)\right)\right\} .
\end{aligned}
$$

Step 7. Similar to Step 3, the "integrated" primal variables are introduced to replace the corresponding discounted values, respectively. Taking $Q L_{i}(t)$ and $Q_{i \max }$ as examples, we have:

$$
Q L_{i}^{* *}=\sum_{t=1}^{4} \delta^{t-1} \cdot Q L_{i}^{*}(t), \quad i \in R,
$$

$$
Q_{i \max }^{* *}=\sum_{t=1}^{4} \delta^{t-1} \cdot Q_{i \max }^{*}, \quad i \in R
$$

Here, all the primal variables have double asterisks in their top right corner. Thus,

$$
\begin{aligned}
\min & \sum_{i \in R}\left\{\Delta t \cdot\left[c_{i} \cdot x_{i} \cdot\left(\mathrm{Q} L_{i}^{* *}-Q_{i \min }^{* * *}\right)-f \cdot K_{i} \cdot \mathrm{Q} E_{i}^{* *} \cdot H_{i}\right]+\beta_{8}^{+} \cdot\left(\mathrm{Q} E_{i}^{* *}-M M \cdot x_{i}\right)\right. \\
& +\beta_{9}^{+} \cdot\left(K_{i} \cdot \mathrm{Q} E_{i}^{* *} \cdot H_{i}-N_{i \max }^{* *}\right)+\beta_{9}^{-} \cdot\left(K_{i} \cdot Q E_{i}^{* *} \cdot H_{i}-x_{i} \cdot N_{i \min }^{* *}\right) \\
& +\beta_{10}^{+} \cdot\left(y_{i}^{* *}-\frac{1}{1-\delta^{4}} \cdot \mathrm{Cap}_{i}^{*}\right)+\beta_{10}^{-} \cdot\left(y_{i}^{* *}-V_{i \min }^{* *} \cdot x_{i}\right)+\beta_{11} \cdot\left(y_{i}^{* *}-y_{i}^{* *}(0)-\Delta t \cdot\left(Q_{i-1}^{* *}+\varepsilon_{i}^{* *}-Q_{i}^{* *}-Q L_{i}^{* *}\right)\right) \\
& +\beta_{12} \cdot\left(Q_{i}^{* *}-Q E_{i}^{* *}-Q A_{i}^{* *}\right)+\beta_{13}^{+} \cdot\left(Q E_{i}^{* *}+Q A_{i}^{* *}-Q F_{i \max }^{* *}\right)+\beta_{13}^{-} \cdot\left(Q E_{i}^{* *}+Q A_{i}^{* *}-Q F_{i \min }^{* *}\right) \\
& \left.+\beta_{14}^{+} \cdot\left(Q L_{i}^{* *}-x_{i} \cdot Q_{i \max }^{* *}-\left(1-x_{i}\right) \cdot Q_{i \min }^{* *}\right)+\beta_{14}^{-} \cdot\left(Q L_{i}^{* *}-Q_{i \min }^{* *}\right)\right\}
\end{aligned}
$$

in which

$$
\operatorname{Cap}_{i}^{*}=\sum_{t=1}^{4} \delta^{t-1} \cdot \operatorname{Cap}_{i}=\frac{1-\delta^{4}}{1-\delta} \cdot \operatorname{Cap}_{i}, \quad i \in R,
$$

$$
y_{i}^{* *}=\sum_{t=1}^{4} \delta^{t-1} \cdot y_{i}^{*}(t)=\sum_{t=1}^{4} \delta^{t-1} \cdot \sum_{\tau=0}^{(T / 4)-1} \delta^{4 \tau} \cdot y_{i}(4 \tau+t) .
$$

and for each $i(\in R), y_{i}^{* *}$ is a discounted value that can be expressed as

From (20), we have 


$$
\delta \cdot y_{i}^{* *}=\sum_{t=1}^{4} \delta^{t} \cdot \sum_{\tau=0}^{(T / 4)-1} \delta^{4 \tau} \cdot y_{i}(4 \tau+t)
$$

Refer to (20), the discounted value $y_{i}^{* *}(0)$ can be denoted as

$$
y_{i}^{* *}(0)=\sum_{t=1}^{4} \delta^{t-1} \cdot y_{i}^{*}(t-1)=\sum_{t=1}^{4} \delta^{t-1} \cdot \sum_{\tau=0}^{(T / 4)-1} \delta^{4 \tau} \cdot y_{i}(4 \tau+t-1) .
$$

From (21) and (22), we have

$$
y_{i}^{* *}(0)=\delta \cdot y_{i}^{* *}+\delta^{0} \cdot y_{i}(0)-\delta^{T} \cdot y_{i}(T) .
$$

Using (23) to replace $y_{i}^{* *}(0)$ in (18), we have the discounted objective as follows:

$$
\begin{aligned}
\min & \sum_{i \in R}\left\{\Delta t \cdot\left[c_{i} \cdot x_{i} \cdot\left(Q L_{i}^{* *}-Q_{i \min }^{* *}\right)-f \cdot K_{i} \cdot Q E_{i}^{* *} \cdot H_{i}\right]\right. \\
& +\beta_{8}^{+} \cdot\left(Q E_{i}^{* *}-M M \cdot x_{i}\right) \\
& +\beta_{9}^{+} \cdot\left(K_{i} \cdot Q E_{i}^{* *} \cdot H_{i}-N_{i \max }^{* *}\right)+\beta_{9}^{-} \cdot\left(K_{i} \cdot Q E_{i}^{* *} \cdot H_{i}-x_{i} \cdot N_{i \min }^{* *}\right) \\
& +\beta_{10}^{+} \cdot\left(y_{i}^{* *}-\frac{1}{1-\delta^{4}} \cdot \frac{1-\delta^{4}}{1-\delta} \cdot \mathrm{Cap}_{i}\right)+\beta_{10}^{-} \cdot\left(y_{i}^{* *}-V_{i \min }^{* *} \cdot x_{i}\right) \\
& +\beta_{11} \cdot\left(y_{i}^{* *}-\delta \cdot y_{i}^{* *}-y_{i}(0)+\delta^{T} \cdot y_{i}(T)-\Delta t \cdot\left(Q_{i-1}^{* *}+\varepsilon_{i}^{* *}-Q_{i}^{* *}-Q L_{i}^{* *}\right)\right) \\
& +\beta_{12} \cdot\left(Q_{i}^{* *}-Q E_{i}^{* *}-Q A_{i}^{* *}\right) \\
& +\beta_{13}^{+} \cdot\left(Q E_{i}^{* *}+Q A_{i}^{* *}-Q F_{i \max }^{* *}\right)+\beta_{13}^{-} \cdot\left(Q E_{i}^{* *}+Q A_{i}^{* *}-Q F_{i \min }^{* *}\right) \\
& \left.+\beta_{14}^{+} \cdot\left(Q L_{i}^{* *}-x_{i} \cdot Q_{i \max }^{* *}-\left(1-x_{i}\right) \cdot Q_{i \min }^{* *}\right)+\beta_{14}^{-} \cdot\left(Q L_{i}^{* *}-Q_{i \min }^{* *}\right)\right\}
\end{aligned}
$$

Similarly, $\delta^{T}$ in (24) can be approximated as zero due to the large $T$. Then, by extracting the relaxed constraints from the above objective function, we have

$\min \sum_{i \in R} \Delta t \cdot\left[c_{i} \cdot x_{i} \cdot\left(Q L_{i}^{* *}-Q_{i \min }^{* *}\right)-f \cdot K_{i} \cdot Q E_{i}^{* *} \cdot H_{i}\right]$

s.t.

$$
\begin{aligned}
& Q E_{i}^{* *} \leq M M \cdot x_{i}, \quad i \in R, \\
& x_{i} \cdot N_{i \min }^{* *} \leq K_{i} \cdot Q E_{i}^{* *} \cdot H_{i} \leq N_{i \max }^{* *}, \quad i \in R, \\
& V_{i \min }^{* *} \cdot x_{i} \leq y_{i}^{* *} \leq \frac{1}{1-\delta} \cdot \operatorname{Cap}_{i}, \quad i \in R,
\end{aligned}
$$$$
(1-\delta) y_{i}^{* *}-y_{i}(0)=\Delta t \cdot\left(Q_{i-1}^{* *}+\varepsilon_{i}^{* *}-Q_{i}^{* *}-Q L_{i}^{* *}\right),
$$$$
i \in R \text {, }
$$

$$
Q_{i}^{* *}=Q E_{i}^{* *}+Q A_{i}^{* *}, \quad i \in R
$$

$$
\begin{aligned}
& Q F_{i \min }^{* *} \leq Q E_{i}^{* *}+Q A_{i}^{* *} \leq Q F_{i \text { max }}^{* *}, \quad i \in R, \\
& Q_{i \text { min }}^{* *} \leq Q L_{i}^{* *} \leq x_{i} \cdot Q_{i \max }^{* *}+\left(1-x_{i}\right) \cdot Q_{i \text { min }}^{* *}, \quad i \in R .
\end{aligned}
$$

Step 8. Combine the above model with each scenario $\widehat{s}(\in \widehat{S})$, which is given in Section 3.1.2 and then integrate with the planning stage of the original MSSP model. Thus, the final DE model, which is a two-stage stochastic program, is developed as (26a) (26k).

$$
\begin{aligned}
& \min \sum_{i \in R} e \cdot \operatorname{Cap}_{i}+\Delta t \cdot \sum_{i \in R}\left(r_{i}^{* *} \cdot \operatorname{Cap}_{i}\right) \\
& +\sum_{\widehat{s} \in \widehat{S}}\left\{\widehat{p^{s}} \cdot \sum_{i \in R} \Delta t \cdot\left[c_{i} \cdot x_{i} \cdot\left(\mathrm{Q} \hat{L_{i}^{\widehat{s}}}-Q_{i \min }^{\prime * * \widehat{s}}\right)-f \cdot K_{i} \cdot \mathrm{Q} E_{i}^{\widehat{s}} \cdot H_{i}\right]\right\},
\end{aligned}
$$

s.t.

$$
R_{\min } \leq \sum_{i \in R} x_{i} \leq R,
$$

$$
\mathrm{Cap}_{i} \leq M M \cdot x_{i}, \quad i \in R,
$$




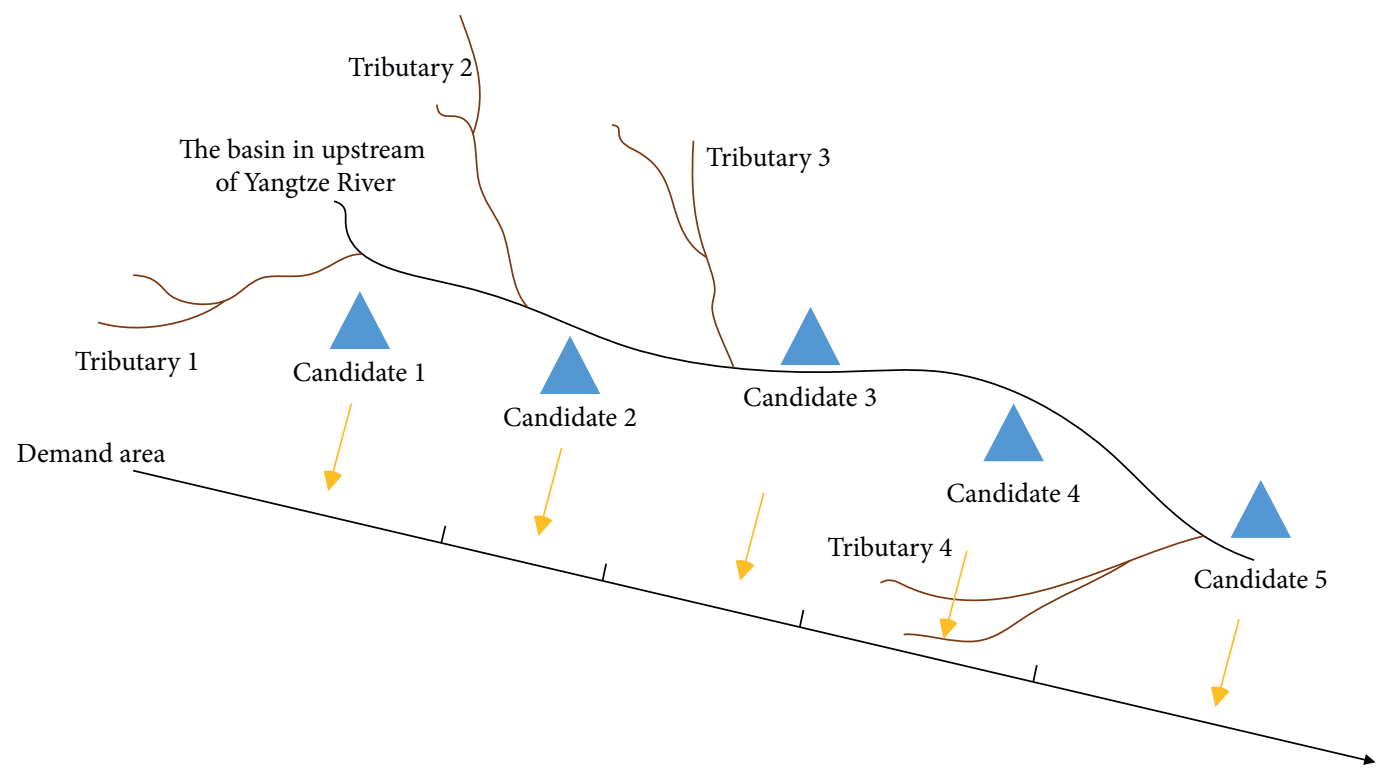

FIgURE 6: Illustration of hydropower station candidate in upstream of Yangtze river.

TABLE 3: Related parameters of hydropower station candidates.

\begin{tabular}{lccccccc}
\hline Candidate $i$ & $K_{i}\left(\mathrm{~kg} /\left(\mathrm{m}^{2} \cdot \mathrm{s}^{2}\right)\right)$ & $N_{i \min }(\mathrm{KW})$ & $N_{i \max }(\mathrm{KW})$ & $H_{i}(\mathrm{~m})$ & $c_{i}\left(\mathrm{RMB} / \mathrm{m}^{3}\right)$ & $r_{i}\left(\mathrm{RMB} /\left(\mathrm{m}^{3} \cdot \mathrm{year}\right)\right)$ & $Q_{i \max }\left(\mathrm{m}^{3} \times 10^{8} / \mathrm{h}\right)$ \\
\hline 1 & 8.4 & 2546250 & 5092500 & 105 & 0.1 & 0.06 & 0.4676 \\
2 & 8.4 & 1506750 & 3013500 & 93 & 0.125 & 0.072 & 0.8550 \\
3 & 8.4 & 1500000 & 3000000 & 90 & 0.15 & 0.05 & 0.5177 \\
4 & 8.4 & 3742500 & 7485000 & 110 & 0.175 & 0.08 & 0.4662 \\
5 & 8.4 & 2625000 & 5250000 & 112 & 0.2 & 0.3 & \\
\hline
\end{tabular}

TABle 4: Minimum storage and limitation of water flows of hydropower station candidates.

\begin{tabular}{lccc}
\hline $\begin{array}{l}\text { Candidate } \\
i\end{array}$ & $\begin{array}{c}V_{\text {imin }} \\
\left(\mathrm{m}^{3} \times 10^{8}\right)\end{array}$ & $\begin{array}{c}Q F_{\text {imin }}\left(\mathrm{m}^{3} \times 10^{8} /\right. \\
\mathrm{h})\end{array}$ & $\begin{array}{c}Q F_{\text {imax }}\left(\mathrm{m}^{3} \times 10^{8} /\right. \\
\mathrm{h})\end{array}$ \\
\hline 1 & 0.0311 & 0.0648 & 1.5732 \\
2 & 0.0213 & 0.0468 & 1.7928 \\
3 & 0.0131 & 0.0432 & 0.4248 \\
4 & 0.0420 & 0.1620 & 3.5568 \\
5 & 0.0115 & 0.0396 & 0.6912 \\
\hline
\end{tabular}

TABLE 5: Random water inflows in each seasonal stage of each hydropower station candidate.

\begin{tabular}{lccccc}
\hline \multirow{2}{*}{ Candidate $i$} & Inflows level & \multicolumn{4}{c}{ Water inflows $\left(\mathrm{m}^{3} \times 10^{7} / \mathrm{h}\right)$} \\
& & Spring & Summer & Autumn & Winter \\
\hline \multirow{2}{*}{1} & $H$ & 1.2846 & 5.7276 & 2.2020 & 0.2031 \\
& $L$ & 0.9867 & 4.3995 & 1.6914 & 0.1560 \\
2 & $H$ & 0.5932 & 1.6312 & 0.6677 & 0.0985 \\
& $L$ & 0.4589 & 1.2617 & 0.5165 & 0.0762 \\
3 & $H$ & 0.5653 & 1.2849 & 0.6739 & 0.0969 \\
& $L$ & 0.4441 & 1.0093 & 0.5294 & 0.0762 \\
4 & $H$ & 0.3645 & 0.5958 & 0.3356 & 0.0930 \\
& $L$ & 0.2575 & 0.4209 & 0.2371 & 0.0657 \\
5 & $H$ & 0.6900 & 1.1642 & 0.5710 & 0.1515 \\
& $L$ & 0.6482 & 1.0936 & 0.5363 & 0.1423 \\
Probability & $H$ & 0.4 & 0.7 & 0.55 & 0.25 \\
& $L$ & 0.6 & 0.3 & 0.45 & 0.75 \\
\hline
\end{tabular}

TABLE 6: Predicted water inflows in each seasonal stage.

\begin{tabular}{lccccc}
\hline & Candidate & \multicolumn{4}{c}{ Stage $(t=1,2,3,4)$} \\
& $i$ & Spring & Summer & Autumn & Winter \\
\hline & 1 & 1.1059 & 5.3292 & 1.9722 & 0.1678 \\
Water inflows & 2 & 0.5126 & 1.5203 & 0.5997 & 0.0817 \\
$\bar{\varepsilon}_{i}(t)\left(\mathrm{m}^{3} \times 10^{7} /\right.$ & 3 & 0.4926 & 1.2202 & 0.6089 & 0.0813 \\
$\mathrm{~h})$ & 4 & 0.3003 & 0.5433 & 0.2912 & 0.0725 \\
& 5 & 0.6649 & 1.1431 & 0.5554 & 0.1446 \\
\hline
\end{tabular}

$$
\begin{aligned}
& Q E_{i}^{\widehat{s}} \leq M M \cdot x_{i}, \quad \widehat{s} \in \widehat{S} ; i \in R, \\
& x_{i} \cdot N_{i \min }^{* *} \leq K_{i} \cdot Q \bar{E}_{i}^{\widehat{s}} \cdot H_{i} \leq N_{i \max }^{* *}, \quad \widehat{s} \in \widehat{S} ; i \in R, \\
& V_{i \min }^{* *} \cdot x_{i} \leq y_{i}^{\widehat{s}} \leq \frac{1}{1-\delta} \cdot \operatorname{Cap}_{i}, \quad \widehat{s} \in \widehat{S} ; i \in R, \\
& (1-\delta) y_{i}^{\widehat{s}}-y_{i}^{\prime}=\Delta t \cdot\left[Q_{i-1}^{\widehat{s}}+\varepsilon_{i}^{* \widehat{s}}-Q_{i}^{\widehat{s}}-Q L_{i}^{\widehat{s}}\right], \widehat{s} \in \widehat{S} ; i \in R \\
& \mathrm{Q}_{i}^{\widehat{s}}=\mathrm{Q} E_{i}^{\widehat{s}}+Q A_{i}^{\widehat{s}}, \quad \widehat{s} \in \widehat{S} ; i \in R, \\
& Q F_{i \min }^{* *} \leq Q \widehat{E_{i}^{s}}+Q A_{i}^{\widehat{s}} \leq Q F_{i \max }^{* *}, \quad \widehat{s} \in \widehat{S} ; i \in R,
\end{aligned}
$$


TABLE 7: Predicted water demands in each seasonal stage.

\begin{tabular}{lccccc}
\hline & Candidate & \multicolumn{4}{c}{ Stage $(t=1,2,3,4)$} \\
& $i$ & Spring & Summer & Autumn & Winter \\
\hline \multirow{2}{*}{ Water } & 1 & 0.1871 & 0.2299 & 0.2196 & 0.1218 \\
demands & 2 & 0.3420 & 0.4204 & 0.4016 & 0.2228 \\
$\bar{Q}_{i \text { min }}(t)$ & 3 & 0.2071 & 0.2546 & 0.2432 & 0.1349 \\
$\left(\mathrm{~m}^{3} \times 10^{6} / \mathrm{h}\right)$ & 4 & 0.1865 & 0.2292 & 0.2189 & 0.1215 \\
& 5 & 0.1321 & 0.1623 & 0.1551 & 0.0860 \\
\hline
\end{tabular}

TABle 8: Estimation gaps of the DE model.

\begin{tabular}{lccc}
\hline \multirow{2}{*}{ Discount factor $\delta$} & \multicolumn{3}{c}{ Planning stages } \\
& $T=4$ & $T=8$ & $T=12$ \\
\hline 0.99 & $25.53 \%$ & $22.51 \%$ & $19.94 \%$ \\
0.95 & $17.89 \%$ & $6.71 \%$ & $-2.99 \%$ \\
0.90 & $8.72 \%$ & $-9.76 \%$ & $-24.32 \%$ \\
0.85 & $-0.51 \%$ & $-23.20 \%$ & $-39.73 \%$ \\
0.80 & $-9.16 \%$ & $-34.34 \%$ & $-50.97 \%$ \\
0.75 & $-17.29 \%$ & $-43.92 \%$ & $-59.59 \%$ \\
0.70 & $-24.72 \%$ & $-51.99 \%$ & $-66.37 \%$ \\
0.65 & $-31.44 \%$ & $-58.62 \%$ & $-71.61 \%$ \\
0.60 & $-37.53 \%$ & $-64.02 \%$ & $-75.64 \%$ \\
\hline
\end{tabular}

Table 9: Average computational time (ms) of both models.

\begin{tabular}{lccc}
\hline Model & $T=4$ & $T=8$ & $T=12$ \\
\hline DE model & 1224.00 & 1206.44 & 1170.22 \\
MSSP model & 300.22 & 3095.56 & 159373.22 \\
\hline
\end{tabular}

TABle 10: Probabilistic distributions of water inflows and demands in each seasonal stage.

\begin{tabular}{lcc}
\hline Stage & Water inflows $\left(p_{1 H}, p_{1 M}\right.$, & Water demands $\left(p_{2 H}\right.$, \\
& $\left.p_{1 L}\right)$ & $\left.p_{2 L}\right)$ \\
\hline Spring & $(0.2,0.4,0.4)$ & $(0.5,0.5)$ \\
Summer & $(0.5,0.4,0.1)$ & $(0.65,0.35)$ \\
Autumn & $(0.3,0.5,0.2)$ & $(0.6,0.4)$ \\
Winter & $(0.1,0.3,0.6)$ & $(0.3,0.7)$ \\
\hline
\end{tabular}

$$
Q_{i \min }^{* \widehat{s}} \leq Q L_{i}^{\widehat{s}} \leq x_{i} \cdot Q_{i \max }^{* *}+\left(1-x_{i}\right) \cdot Q_{i \min }^{* \widehat{s}}, \quad \widehat{s} \in \widehat{S} ; i \in R,
$$

$$
\operatorname{Cap}_{i}, y_{i}^{\widehat{s}}, Q L_{i}^{\widehat{s}}, Q E_{i}^{\widehat{s}}, \widehat{Q_{i}^{s}}, Q A_{i}^{\widehat{s}} \geq 0, \quad x_{i} \in\{0,1\}, i \in R ; \widehat{s} \in \widehat{S}
$$

in which the discounted value of water inflows and demands $\varepsilon_{i}^{* * \widehat{s}}$ and $Q_{i \min }^{* * \widehat{s}}(i \in R)$ can be obtained similar to the way used in formula (3). Besides, $r_{i}^{* *}$ in (26a) is an integrated variable:

$$
r_{i}^{* *}=\sum_{t=1}^{4} \delta^{t-1} \cdot r_{i}^{*}(t), \quad i \in R
$$

Moreover, for simplicity, the double asterisks of the decision variables are omitted.
After transformation, the planning decisions $x_{i}$ and Cap remain unchanged, while the operational stages are aggregated. Therefore, the proposed approach not only keeps the future impacts but also reduces the solving complexity.

\section{Experimental Study}

To validate the applicability of our proposed approach, we give the data of a case in Section 4.1 at first. Then, based on this case, we compare our approach with the MSSP model in the small-scale setting in Section 4.2. Furthermore, we apply our approach to an overlong-term case in Section 4.3 and finally implement sensitivity analysis in Section 4.4.

4.1. Description of the Case. Yangtze River flows through 11 provinces in China with a drainage area of 1.8 million square kilometers. The whole river can be divided into the upstream, midstream, and downstream, among which the basin with a length of $1030 \mathrm{~km}$ in upstream is studied here. According to Wang et al. [45], five hydropower station candidates could be considered in this basin. Its schematization is shown in Figure 6 which also involves the main tributaries.

Among the five hydropower station candidates, at least two of them should be chosen in the plan. Unit construction fee $(e)$ and unit profit of power generation $(f)$ in Table 1 are set to be $5.09\left(\mathrm{RMB} / \mathrm{m}^{3}\right)$ and $0.25(\mathrm{RMB} / \mathrm{KWh})$, respectively [46]. Related parameters regarding five candidates are displayed in Table 3. Once the hydropower station candidate is chosen, the planned lowest water storage and upper and lower limit of water discharge are shown in Table 4 . The initial water flows from upstream to each hydropower station candidate is set to be 0.2141, 0.0988, 0.0942, 0.0429, and $0.1080\left(\mathrm{~m}^{3} \times 10^{8} / \mathrm{h}\right)$, respectively. And the initial water storage of each chosen hydropower station is assumed as the twice of the lowest water storage.

4.2. Comparison with the MSSP Model by Small-Scale Cases. The developed DE model is an approximate version of the MSSP model (1). In this section, we aim to evaluate the performance of our approach by comparing the planning decisions as well as the operational performance with these of the MSSP model. Due to the curse of dimensionality faced by the MSSP model, we consider small-scale cases based on Section 4.1.

4.2.1. Parameters in Small-Scale Experiments. To limit the dimensionality here, we set the planning horizon as 1,2 , and 3 years, respectively. According to the seasonal features of which, one year is divided into four stages: spring (March to May), summer (June to August), autumn (September to November), and winter (December to February). Then, each stage has $2160(h)$ (i.e., $\Delta t$ ). Thus, the number of operational stages $T$ is 4,8 , and 12 , respectively. Besides, we merely consider the uncertainty caused by water inflows. Two kinds of stochastic outside water inflows $(H, L)$, as well as the 


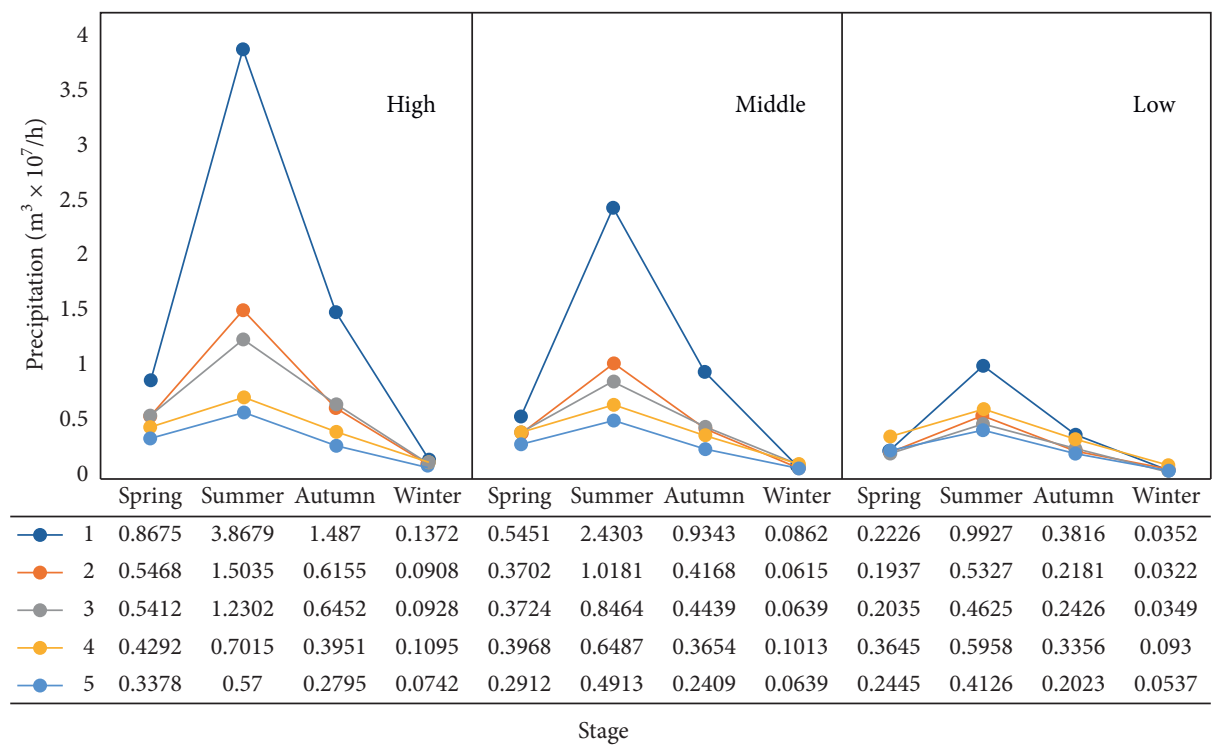

FIGURE 7: High, middle, low-level precipitation in each seasonal stage of each hydropower station candidate.

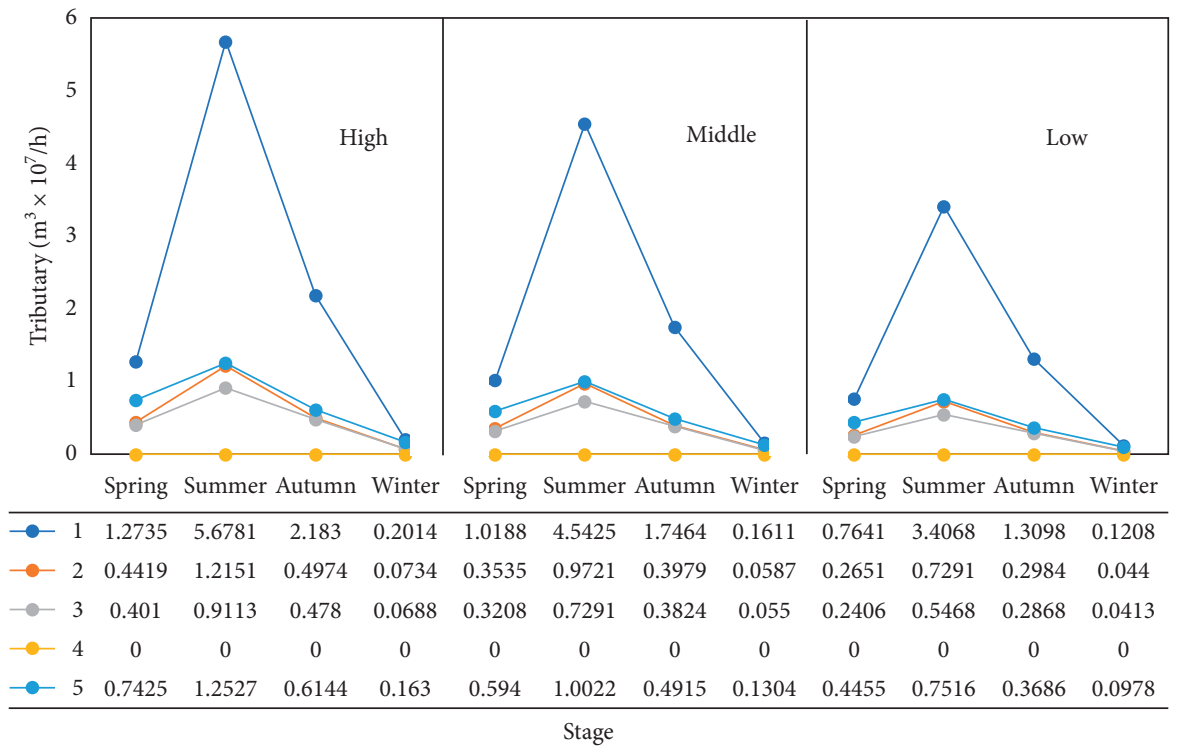

Figure 8: High, middle, low-level tributary in each seasonal stage of each hydropower station candidate.

corresponding probabilities $\left(p_{1 H}, p_{1 L}\right)$, in each seasonal stage are given in Table 5 . The constant water inflows and water demands used in the simplification are displayed in Tables 6 and 7, respectively. The acceptance level of the water spill is assumed as twice of the demands.

Because the random inflows fluctuate with seasons, we assume the water inflows of each candidate take the same uncertain level in each season. Thus, for the MSSP model, $16\left(=2^{4}\right), 256\left(=2^{8}\right)$, and $4096\left(=2^{12}\right)$ stagewise scenarios are constructed, respectively. Furthermore, according to the generation and simplification process of the scenario set displayed in Section 3.1, 16 scenarios are generated for the two-stage DE model. Assume that spring is the first operational season. The discount factor $\delta$ used in the DE model is set to be $0.99,0.95, \ldots, 0.60$, respectively.
4.2.2. Computational Results. We optimally solve the DE model and the MSSP model by IBM ILOG CPLEX 12.6.3 on a PC with $8 \mathrm{~GB}$ memory and a CPU at $2.5 \mathrm{GHz}$.

It is found that the DE model can generate the same planning results with the MSSP model for all these smallscale cases. Besides, in terms of the operations, the DE model considers the multistage operational impacts by the aggregated way. Hence, it is interesting to observe how accurate the DE model is in the estimation of the future operational performance of the cascade hydropower system. We use DE_2nd and MSSP_2nd to denote the operational performance generated by the DE model and the MSSP model, respectively. Table 8 displays the estimation gap (=(DE_2nd-MSSP_2nd)/MSSP_2nd) under different discount factors. 


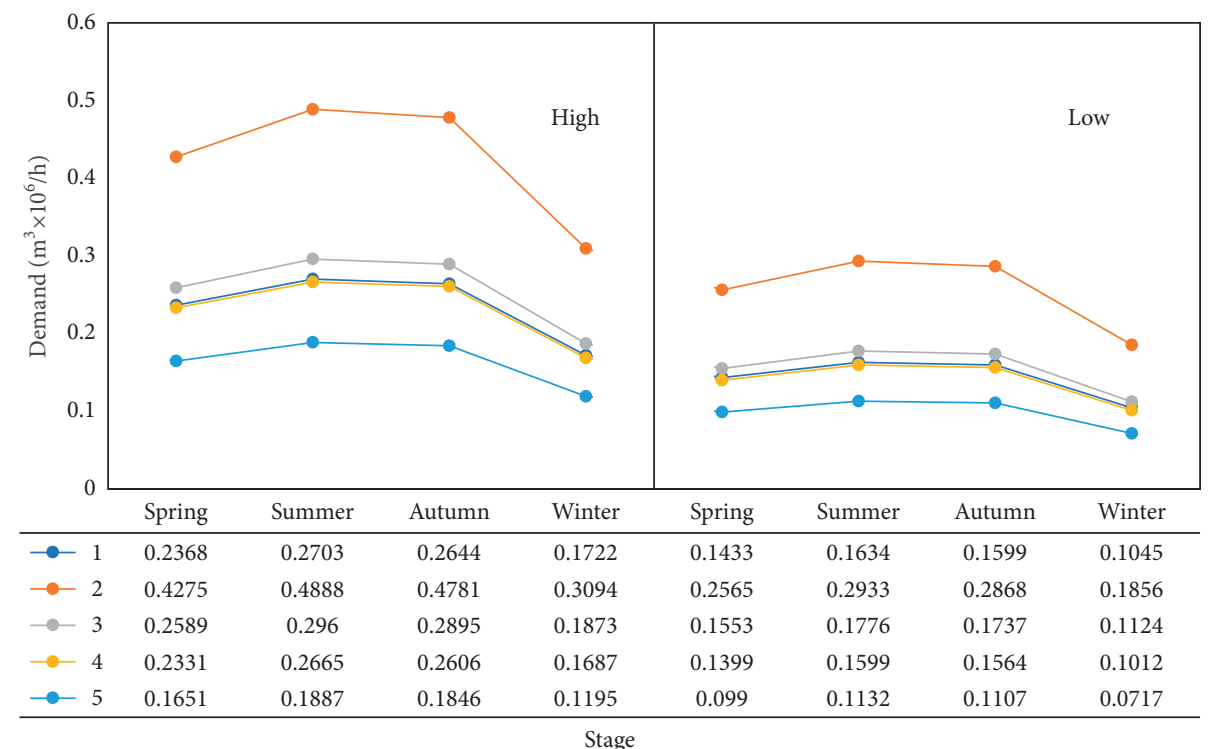

FIgURE 9: High, low-level water demand in each seasonal stage of each hydropower station candidate.

TABle 11: Predicted water inflows and demands in each seasonal stage.

\begin{tabular}{|c|c|c|c|c|c|}
\hline & \multirow{2}{*}{ Candidate $i$} & \multicolumn{4}{|c|}{ Stage $(t=1,2,3,4)$} \\
\hline & & Spring & Summer & Autumn & Winter \\
\hline \multirow{5}{*}{ Water inflows $\bar{\varepsilon}_{i}(t)\left(\mathrm{m}^{3} \times 10^{7} / \mathrm{h}\right)$} & 1 & 1.4484 & 8.0021 & 2.7796 & 0.2017 \\
\hline & 2 & 0.6708 & 2.2816 & 0.8445 & 0.0982 \\
\hline & 3 & 0.6433 & 1.8019 & 0.8559 & 0.0975 \\
\hline & 4 & 0.3904 & 0.6698 & 0.3683 & 0.0971 \\
\hline & 5 & 0.8462 & 1.6252 & 0.7486 & 0.1729 \\
\hline \multirow{5}{*}{ Water demands $\bar{Q}_{i \min }(t)\left(\mathrm{m}^{3} \times 10^{6} / \mathrm{h}\right)$} & 1 & 0.1871 & 0.2299 & 0.2196 & 0.1218 \\
\hline & 2 & 0.3420 & 0.4204 & 0.4016 & 0.2228 \\
\hline & 3 & 0.2071 & 0.2546 & 0.2432 & 0.1349 \\
\hline & 4 & 0.1865 & 0.2292 & 0.2189 & 0.1215 \\
\hline & 5 & 0.1321 & 0.1623 & 0.1551 & 0.0860 \\
\hline
\end{tabular}

TABLE 12: Planning decisions and the cost/benefit.

\begin{tabular}{lcccc}
\hline Candidate $i$ & $\mathrm{Cap}_{i}\left(\mathrm{~m}^{3} \times 10^{8}\right)$ & Operational cost $\left(\mathrm{RMB} \times 10^{8}\right)$ & Penalty cost $\left(\mathrm{RMB} \times 10^{8}\right)$ & Benefit $\left(\mathrm{RMB} \times 10^{8}\right)$ \\
\hline 1 & 0.214 & 0.063 & 347.191 & 550.002 \\
4 & 0.043 & 0.017 & 0 & 808.401 \\
5 & 0.108 & 0.053 & 0 & 567.013 \\
\hline
\end{tabular}

TABLE 13: Operational decisions under scenario 16.

\begin{tabular}{|c|c|c|c|c|c|}
\hline Candidate $i$ & 1 & 2 & 3 & 4 & 5 \\
\hline$Q L_{i}^{16}\left(\mathrm{~m}^{3} \times 10^{8} / \mathrm{h}\right)$ & 2.154 & 0.066 & 0.040 & 0.036 & 0.026 \\
\hline $\mathrm{QE}_{i}^{16}\left(\mathrm{~m}^{3} / \mathrm{h}\right)$ & 115476 & 0 & 0 & 162013 & 111607 \\
\hline$Q A_{i}^{16}\left(\mathrm{~m}^{3} \times 10^{8} / \mathrm{h}\right)$ & 4.690 & 6.714 & 8.496 & 9.246 & 11.014 \\
\hline$y_{i}^{16^{2}}\left(\mathrm{~m}^{3} \times 10^{8}\right)$ & 4.282 & 0 & 0 & 0.858 & 2.161 \\
\hline$Q_{i}^{16}\left(\mathrm{~m}^{3} \times 10^{8} / \mathrm{h}\right)$ & 4.691 & 6.714 & 8.496 & 9.248 & 11.015 \\
\hline
\end{tabular}

TABle 14: Operational decisions under scenario 1281.

\begin{tabular}{lccccc}
\hline Candidate $i$ & 1 & 2 & 3 & 4 \\
\hline$Q L_{i}^{1281}\left(\mathrm{~m}^{3} \times 10^{8} / \mathrm{h}\right)$ & 0.874 & 0.073 & 0.044 & 0.040 & 0.028 \\
$\mathrm{Q} E_{i}^{1281}\left(\mathrm{~m}^{3} / \mathrm{h}\right)$ & 115476 & 0 & 0 & 8.496 & 162013 \\
$\mathrm{Q} A_{i}^{1281}\left(\mathrm{~m}^{3} \times 10^{8} / \mathrm{h}\right)$ & 5.171 & 6.936 & 0 & 9.219 & 111607 \\
$y_{i}^{1281}\left(\mathrm{~m}^{3} \times 10^{8}\right)$ & 4.282 & 0 & 8.496 & 0.858 & 10.847 \\
$Q_{i}^{1281}\left(\mathrm{~m}^{3} \times 10^{8} / \mathrm{h}\right)$ & 5.172 & 6.936 & 9.221 & 0.230 \\
\hline
\end{tabular}


TABLE 15: Corresponding cost terms and benefit.

\begin{tabular}{|c|c|c|c|c|c|}
\hline $\begin{array}{l}\text { Operational } \\
\text { stages }\end{array}$ & $\begin{array}{c}\text { Candidate } \\
i\end{array}$ & $\begin{array}{l}\text { Total performance } \\
\quad\left(\mathrm{RMB} \times 10^{8}\right)\end{array}$ & $\begin{array}{l}\text { Operational cost } \\
\left(\mathrm{RMB} \times 10^{8}\right)\end{array}$ & $\begin{array}{l}\text { Penalty cost } \\
\left(\mathrm{RMB} \times 10^{8}\right)\end{array}$ & $\begin{array}{c}\text { Power generation } \\
\quad\left(\mathrm{RMB} \times 10^{8}\right)\end{array}$ \\
\hline \multirow{4}{*}{$T=40$} & 1 & 471.396 & 0.063 & 77.453 & 550.002 \\
\hline & 4 & 808.165 & 0.017 & 0 & 808.401 \\
\hline & 5 & 566.410 & 0.053 & 0 & 567.013 \\
\hline & Total & 1845.971 & 0.134 & 77.453 & 1925.416 \\
\hline \multirow{4}{*}{$T=80$} & 1 & 236.358 & 0.063 & 312.491 & 550.002 \\
\hline & 4 & 808.165 & 0.017 & 0 & 808.401 \\
\hline & 5 & 566.410 & 0.053 & 0 & 567.013 \\
\hline & Total & 1610.933 & 0.134 & 312.491 & 1925.416 \\
\hline \multirow{4}{*}{$T=120$} & 1 & 205.604 & 0.063 & 343.245 & 550.002 \\
\hline & 4 & 808.165 & 0.017 & 0 & 808.401 \\
\hline & 5 & 566.410 & 0.053 & 0 & 567.013 \\
\hline & Total & 1580.179 & 0.134 & 343.245 & 1925.416 \\
\hline \multirow{4}{*}{$T=160$} & 1 & 201.653 & 0.063 & 347.196 & 550.002 \\
\hline & 4 & 808.165 & 0.017 & 0 & 808.401 \\
\hline & 5 & 566.410 & 0.053 & 0 & 567.013 \\
\hline & Total & 1576.228 & 0.134 & 347.196 & 1925.416 \\
\hline \multirow{4}{*}{$T=200$} & 1 & 201.150 & 0.063 & 347.699 & 550.002 \\
\hline & 4 & 808.165 & 0.017 & 0 & 808.401 \\
\hline & 5 & 566.410 & 0.053 & 0 & 567.013 \\
\hline & Total & 1575.725 & 0.134 & 347.699 & 1925.416 \\
\hline
\end{tabular}

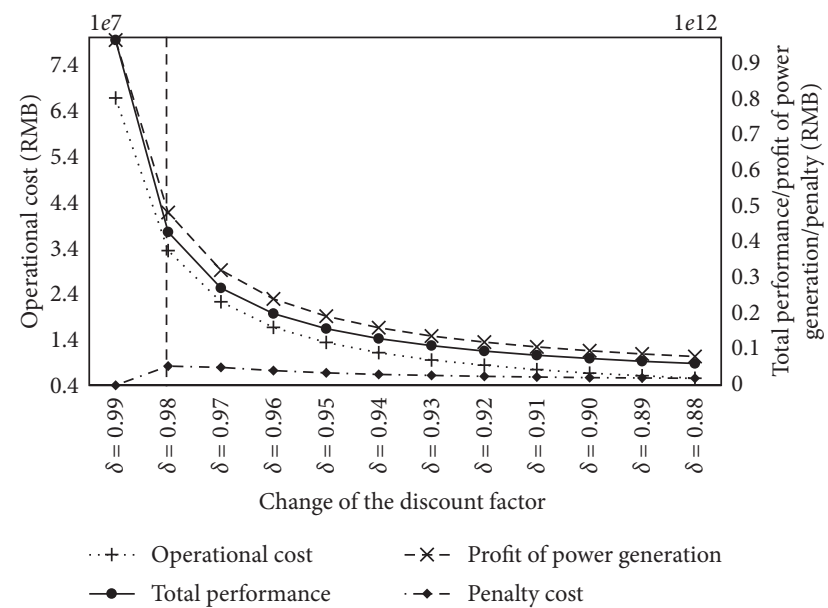

FIGURE 10: Cost and profit of power generation under the different discount factors.

It is found that the operational performance generated by the DE model significantly varies with the discount factors. Specifically, when $\delta$ is large, more future impacts would be taken into account which results in the overestimation. Hence, the estimation gap is positive. With the decrease of $\delta$, less future performance would be discounted. Thus, the estimation gap tends to be negative gradually. It also means that the DE model can generate accurate estimation of the future operational performance by choosing the appropriate discount factor.

Moreover, the computational time of the DP model outperforms that of the MSSP model greatly especially when the horizon is large, as shown in Table 9.

In summary, the DP model can yield the same location decisions with the MSSP model and the accurate estimation

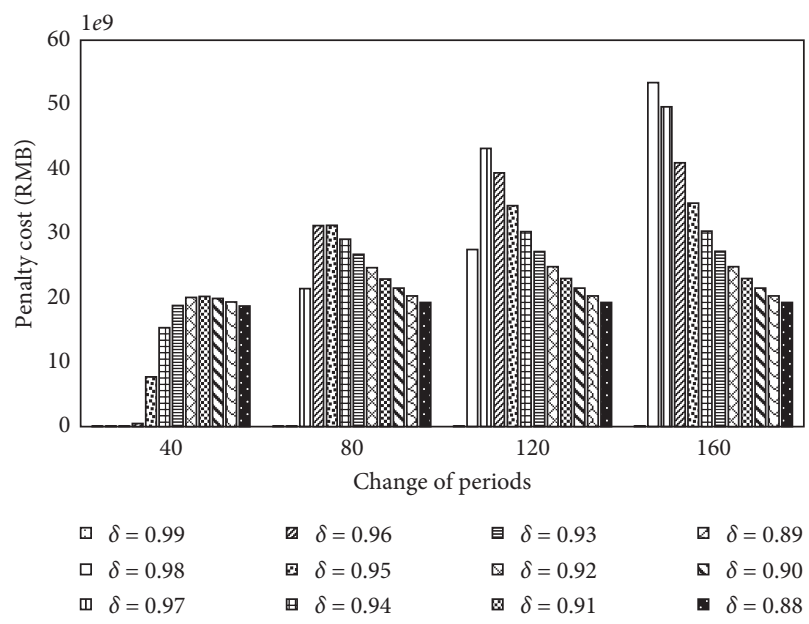

Figure 11: Penalty cost under the different discount factors and planning horizons.

of the future operational performance by choosing a suitable discount factor, while less calculational time is required.

4.3. Real Case Study. Section 4.2 validates the effectiveness of our proposed DE model in the small-scale setting. In this section, we further apply our approach to a large-scale case to illustrate its applicability.

4.3.1. Parameters in the Large-Scale Case. Based on the case given in Section 4.1, we consider the 40-year planning horizon (i.e., $T=160)$. Three kinds of stochastic water inflows $(H, M$, and $L)$ and two kinds of stochastic water demands $(H, L)$ are taken into account in each seasonal stage. The corresponding probabilities of two random variables are $\left(p_{1 H}, p_{1 M}, p_{1 L}\right)$ and $\left(p_{2 H}, p_{2 L}\right)$ as shown in Table 10. Refer to 


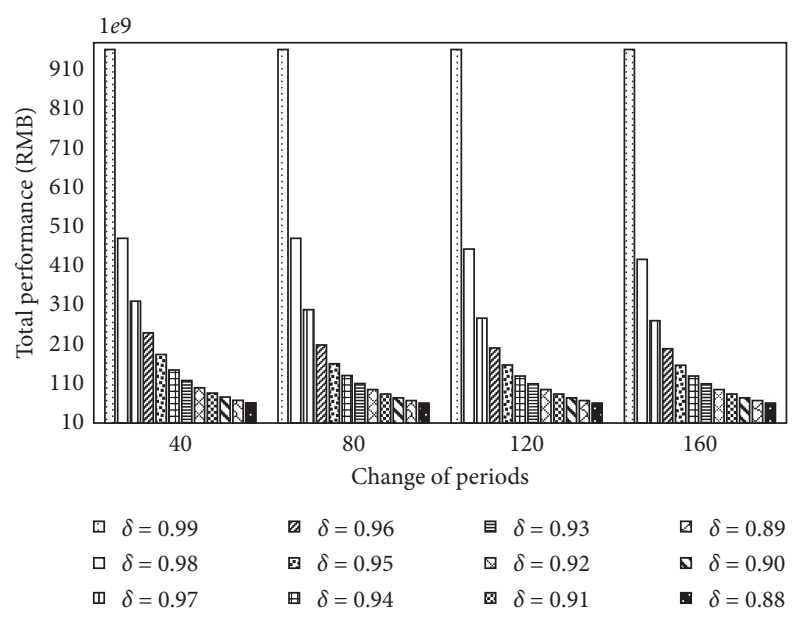

FIGURE 12: Total performance under the different discount factors and planning horizons.

Wang et al. [47] and Yang and Zhang [48]. Uncertain water inflows which mainly include precipitation and water from main tributaries (drainage areas larger than ten thousand square kilometers) are given in Figures 7 (precipitation) and 8 (tributary), respectively. Random demands are given in Figure 9. Besides, the predicted water inflows and demands used in the DE model are shown in Table 11, and the random acceptance level of water spill is also assumed as twice of the corresponding demands.

Thus, the number of stagewise scenarios in the MSSP model is up to $6^{160}\left(\approx 3.193 \times 10^{124}\right)$, which is impossible to handle exactly. By the DE model, the number of scenarios is reduced to 1296 . The discount factor $\delta$ is set to be 0.95 here.

4.3.2. Computational Results of the DE Model. The optimal solution of the DE model can be obtained by within 2047 (ms) by IBM ILOG CPLEX 12.6.3, in which Candidates 1, 4, and 5 are selected. Table 12 shows the capacity decisions and the operational performance, including the operational cost, the penalty cost, and the profit of power generation, of each selected candidate. The total performance value including the planning stage and the operational stages is $1.576 \times 10^{11}$ (RMB).

We further observe two extreme scenarios. One is scenario 16 (high-level inflows and low-level demands in all four seasons), and another scenario is 1281 (low-level inflows and high-level demands in all four seasons). The corresponding scenario-dependent operational results of five hydropower station candidates under two scenarios are shown in Tables 13 and 14, respectively.

As shown in Tables 13 and 14, the daily scheduling strategies would be adjusted according to different scenarios. First, in terms of loss flow $(Q L)$, when scenario 16 happens, the value is relatively high in hydropower station 1 to prevent the flood (see Table 13). While if scenario 1281 occurs, the loss flow merely satisfies demand (see Table 14). Then, regarding water storage $(y)$, the value is high in hydropower station 5 under scenario 16 due to the high-level inflows and the low-level demands. However, scenario 1281, which represents the low-level inflows and the high-level demands, requires hydropower stations to utilize their storage efficiently and then results in low water storage. Furthermore, in the computational results, the scheduling strategies would ensure that the values of water discharge $(Q)$ are between the upper and lower limits in both scenarios, making it capable of preventing flood and drainage. Notice that the water discharges of power generation under two scenarios are the same and the upper limit of output has been reached under both scenarios. Thus, the scheduling strategies can always pursue maximum benefits of power generation under different scenarios. In such a way, the proposed DE model can optimize the global performance in the uncertain setting.

4.4. Sensitivity Analysis. In this section, we carry out the sensitivity analysis to reveal how critical parameters influence the strategic decisions and the performance of the cascade hydropower stations. Because one of the merits is to handle overlong stochasticity by the idea of aggregation, two natural concerns are how long and how much are discounted. Here, we focus on the planning horizon $(T)$ and the discount factor $(\delta)$.

4.4.1. Sensitivity Analysis of the Planning Horizon. The planning horizon $(T)$ is one of the main factors causing the curse of dimensionality $[19,41]$. The DE model aggregates the multiple operational stages, but $T$ would influence the results of the model transformation. Hence, we focus on the length of the planning horizon here. Considering the longlasting effects of the location decisions, we set the length of the planning horizon as $10,20,30,40$, and 50 years, respectively. That is, we consider $T=40,80,120,160$, and 200 . Other parameters are the same as those in Section 4.3.

After calculation, it is found that the decisions of location and capacity under different $T$, which are the planning results we care about in our study, are the same as these of $T=160$ (see Table 12). It means these planning horizons would not alter the strategic decisions of the DE model.

Furthermore, the cost terms and the profit of power generation are displayed in Table 15. It is found that the operational cost and the profit of power generation remain unchanged while the penalty approaches to be stable with the increase of $T$. Specifically, the operational cost is the capacity-related cost within the planning horizon. In the DE model, the impacts of the capacity-related cost are aggregated by $\delta^{T}$, respectively. When $T$ is extremely large, $\delta^{T}$ can be approximated as zero, see (12l) and (12n). Hence, the operational cost keeps unchanged. Besides, to calculate the benefit of the power generation, it is found that the maximum output is reached in our cases. Thus, after aggregation by $\delta^{T}$, the benefit of the power generation also remains constant. Moreover, the penalty of hydropower station 4 and 5 are zero because the loss flows are smaller than the corresponding penalty thresholds. However, $T$ would influence $y_{i}^{* *}($ see $(20))$ at first and then the loss flow by constraints (25e). Hence, the penalty of hydropower station 1 would increase gradually until coming to be stable. 
Based on the above three terms, with the increase of $T$, the total performance also shows a trend of increasing first and then approaches to be stable. This result coincides with the essence of the DE model. Specifically, the farther the future is, the fewer the impacts would be taken into account. Thus, the results would close to be stable finally. It is also in accord with the decision-making in the real-world, i.e., the coming future would be put more emphasis than the superfar future. Hence, when $T$ is large enough, the calculational results of the DE model would tend to be stable. It means that the DE model could describe the steady results of long-term stochastic operations. Thus, for the decisionmakers (DMs), it is not necessary to increase the planning horizon infinitely.

4.4.2. Sensitivity Analysis of the Discount Factor. Discount factor $(\delta)$ is an exogenous variable introduced by the DE model to help to reduce dimensionality. In Section 4.2 , we have shown that the DE model can generate accurate operational performance estimation by selecting appropriate $\delta$. In this section, we further explore the impacts of the discount factor in the large-scale setting. We vary it as 0.99 , $0.98, \ldots, 0.88$. Other parameters are the same as those of Section 4.3.

The calculational results show the discount factors in the value range above will not influence the final strategic decisions. Besides, the operational cost, the penalty cost, the profit of power generation, and the total performance under different discount factors are shown in Figure 10. Firstly, it is shown that, with the decrease of the discount factor, both the operational cost and the profit decline with a decreasing speed since they are discounted more. Moreover, in terms of the penalty cost, it remains zero when $\delta$ is large (0.99). This is because the discounted penalty threshold also decreases but is still larger than the loss flow. Then, the penalty cost increases significantly with the decline of $\delta$ to 0.98 . The reason is that the discounted penalty threshold begins to be smaller than the loss flow, leading to the larger penalty. Finally, with its continuous decrease (less than 0.98), $\delta$ dominates the penalty term which makes the aggregated penalty smaller.

These results indicate different discount factors will not influence the location decisions but result in the variation of the operational performance. Hence, the DMs should carefully choose the discount factor if they want to get an accurate estimation of the operational performance.

4.4.3. Mutual Effects of Two Factors. We further investigate the mutual impacts of two factors on decisions and objective terms. We set $T$ as $40,80,120,160$, and 200 and $\delta$ as 0.99 , $0.98, \ldots, 0.88$ and then calculate the model under each combination of two kinds of parameters.

From the calculational results, we find that the locations and capacities of hydropower stations remain unchanged under the different combinations of planning horizon and discount factor. Given a $\delta$, the operational cost and the profit of power generation will not be influenced by the change of $T$, which is similar to Table 15 . Hence, we focus on the penalty and the total operational performance.
Figure 11 shows the varying pattern of the penalty with the decrease of $\delta$ under each T. Specifically, the penalty increases first and then decreases. The reason is the same as that in Figure 10. However, with the increase of $T$, the peak point would appear earlier. It is because bigger $T$ can bring significant discounting effects due to $\delta^{T}$.

Furthermore, we show the change of the total performance in Figure 12. The variations under different $T$ are very similar. Specifically, with the decline of $\delta$, the total performance gradually decreases and finally tends to be stable. Besides, as $\delta$ becomes smaller, the total performance would reach its steady state within a shorter planning horizon, specifically, around 120 periods when $\delta=0.96$ and around merely 40 periods when $\delta=0.90$. Thus, we conclude that when the low $\delta$ is considered, the DMs could only consider a relatively shorter planning horizon since the impacts in the long-term future would be significantly discounted.

\section{Conclusions and Future Research Directions}

The integrated planning of cascade hydropower stations, which takes the operational strategies under future uncertainty into account, can bring global benefit. An MSSP model is developed to deal with the location and capacity decisions of cascade hydropower stations with the consideration of the expected long-term performance. To address the computational issue caused by the overlong stochasticity, a dimensionality reduction approach based on $\mathrm{DE}$ is designed to transform the MSSP model into a two-stage stochastic programming model, in which future impacts remain by aggregating the operational stages together. Thus, the optimization of the large-scale hydropower stations could be addressed regardless of the number of operational stages.

Experiments based on Yangtze River in China are implemented. First, by the comparison with the MSSP model under the small-sized cases, it is found that our approach can obtain the same planning results and accurate estimation for future operational performance with a short computational time. Then, a large-scale case is studied to show how the approach is applied to solve a practical problem. Moreover, to evaluate the influence of critical parameters in the proposed DE model, sensitivity analysis is conducted based on the planning horizon and the discount factor. The results reveal that our model is robust to different parameter settings to obtain stable location-related planning results, while the estimation of the future performance would be influenced by the planning horizon and the discount factor. The corresponding observations and implications are also obtained from the results.

In the future, we can extend our study in the following aspects. First, the discount factor plays an important role in our study. Specifically, it not only discounts the future impacts to get the so-called net present value but also has the internal relationship between the dual multipliers. Thus, it influences the accuracy of the future estimation of the DE model. In this paper, we follow the way of Grinold [42] and King and Wallace [43] to give the discount factor directly. But, how to select the appropriate discount factor to get 
better performance estimation should be considered in the future study. It is a hard issue but deserves further effort. Second, the minimum installed power and the nonlinearity, like the stage-storage curve, are ignored in this paper. The consideration of these requirements and constraints could help to improve the applicability of our approach to practical problems, which leaves certain spaces for future study. Finally, in this paper, the impacts of time-varying stochastic operations are considered in the form of expected value. Another popular way is to formulate the objective of Valueat-Risk or Conditional Value-at-Risk to incorporate the DMs' risk attitudes. Then, is it possible to utilize the characteristics of Value-at-Risk or Conditional Value-atRisk to facilitate our scenario tree reduction? It is another interesting research direction.

\section{Data Availability}

All data included in this paper are available upon request by contact with the corresponding author.

\section{Conflicts of Interest}

The authors declare that there are no conflicts of interest regarding the publication of this paper.

\section{Acknowledgments}

The authors wish to express their gratitude to the five anonymous referees for their constructive criticism, which led to substantial improvements of the paper. This research was partly supported by the National Natural Science Foundation of China (Grant no. 71971053, 71832001, 71572033) and the MOE (Ministry of Education in China) Project of Humanities and Social Sciences (Grant no. 18YJA630129).

\section{References}

[1] A. Afshar, F. Sharifi, and M. R. Jalali, "Non-dominated archiving multi-colony ant algorithm for multi-objective optimization: application to multi-purpose reservoir operation," Engineering Optimization, vol. 41, no. 4, pp. 313-325, 2009.

[2] P. Fraenkel, O. Paish, V. Bokalders, A. Harvey, and A. E. Brown, Micro-Hydro Power: A Guide for Development Workers, IT Publications Ltd., London, UK, 1999.

[3] G. Eiger and U. Shamir, "Optimal operation of reservoirs by stochastic programming," Engineering Optimization, vol. 17, no. 4, pp. 293-312, 1991.

[4] A. Gjelsvik, B. Mo, and A. Haugstad, "Long- and mediumterm operations planning and stochastic modelling in hydrodominated power systems based on stochastic dual dynamic programming," in Handbook of Power Systems I; Energy Systems; Section 2, Springer, Berlin, Germany, 2010.

[5] C. Cheng, S. Wang, K.-W. Chau, and X. Wu, "Parallel discrete differential dynamic programming for multireservoir operation," Environmental Modelling \& Software, vol. 57, pp. 152-164, 2014.

[6] C. Wang, J. Zhou, P. Lu, and L. Yuan, "Long-term scheduling of large cascade hydropower stations in Jinsha river, China," Energy Conversion and Management, vol. 90, no. 15, pp. 476-487, 2015.
[7] Y. Xu and Y. Mei, "A modified water cycle algorithm for longterm multi-reservoir optimization," Applied Soft Computing, vol. 71, pp. 317-332, 2018.

[8] D. G. Larentis, W. Collischonn, F. Olivera, and C. E. M. Tucci, "GIS-based procedures for hydropower potential spotting," Energy, vol. 35, no. 10, pp. 4237-4243, 2010.

[9] B. C. Kusre, D. C. Baruah, P. K. Bordoloi, and S. C. Patra, "Assessment of hydropower potential using GIS and hydrological modeling technique in Kopili river basin in Assam (India)," Applied Energy, vol. 87, no. 1, pp. 298-309, 2010.

[10] B. Serpoush, M. Khanian, and A. Shamsai, "Hydropower plant site spotting using geographic information system and a MATLAB based algorithm," Journal of Cleaner Production, vol. 152, pp. 7-16, 2017.

[11] A. Z. Zaidi and M. Khan, "Identifying high potential locations for run-of-the-river hydroelectric power plants using GIS and digital elevation models," Renewable and Sustainable Energy Reviews, vol. 89, pp. 106-116, 2018.

[12] J. Hosnar and A. Kovač-Kralj, "Mathematical modelling and MINLP programming of a hydro system for power generation," Journal of Cleaner Production, vol. 65, no. 4, pp. 194201, 2014.

[13] C. Loannidou and J. R. O’Hanley, "Eco-friendly location of small hydropower," European Journal of Operational Research, vol. 264, pp. 907-918, 2018.

[14] D. A. G. Vieira, L. S. M. Guedes, A. C. Lisboa, and R. R. Saldanha, "Formulations for hydroelectric energy production with optimality conditions," Energy Conversion and Management, vol. 89, pp. 781-788, 2015.

[15] C. T. Cheng, J. J. Shen, and X. Y. Wu, "Short-term scheduling for large-scale cascaded hydropower systems with multivibration zones of high head," Journal of Water Resource Planning and Management, vol. 138, no. 3, pp. 257-267, 2012.

[16] C. Li, J. Zhou, S. Ouyang, X. Ding, and L. Chen, "Improved decomposition-coordination and discrete differential dynamic programming for optimization of large-scale hydropower system," Energy Conversion and Management, vol. 84, pp. 363-373, 2014.

[17] X. Li, J. Wei, T. Li, G. Wang, and W. W.-G. Yeh, “A parallel dynamic programming algorithm for multi-reservoir system optimization," Advances in Water Resources, vol. 67, no. 4, pp. 1-15, 2014b.

[18] Z.-K. Feng, W.-J. Niu, C.-T. Cheng, and S.-L. Liao, "Hydropower system operation optimization by discrete differential dynamic programming based on orthogonal experiment design," Energy, vol. 126, pp. 720-732, 2017.

[19] Z.-K. Feng, W.-J. Niu, C.-T. Cheng, and X.-Y. Wu, "Optimization of hydropower system operation by uniform dynamic programming for dimensionality reduction," Energy, vol. 134, pp. 718-730, 2017.

[20] C. Cheng, J. Shen, X. Wu, and K.-W. Chau, "Short-term hydroscheduling with discrepant objectives using multi-step progressive optimality algorithm," JAWRA Journal of the American Water Resources Association, vol. 48, no. 3, pp. 464-479, 2012.

[21] Z.-K. Feng, W.-J. Niu, and C.-T. Cheng, "Optimizing electrical power production of hydropower system by uniform progressive optimality algorithm based on two-stage search mechanism and uniform design," Journal of Cleaner Production, vol. 190, pp. 432-442, 2018.

[22] W.-J. Niu, Z.-K. Feng, C.-T. Cheng, and X.-Y. Wu, "A parallel multi-objective particle swarm optimization for cascade hydropower reservoir operation in southwest China," Applied Soft Computing, vol. 70, pp. 562-575, 2018. 
[23] G. Zhang, Y. Wu, F. Zhang, and X. Liu, "Application of adaptive quantum particle swarm optimization algorithm for optimal dispatching of cascaded hydropower stations," in Intelligent Computing Theories and Applications, Lecture Notes in Computer Science, Springer, Berlin, Germany, 2012.

[24] J. Zhang, Q. Tang, Y. Chen, and S. Lin, "A hybrid particle swarm optimization with small population size to solve the optimal short-term hydro-thermal unit commitment problem," Energy, vol. 109, pp. 765-780, 2016.

[25] S. Ouyang, J. Zhou, C. Li, X. Liao, and H. Wang, "Optimal design for flood limit water level of cascade reservoirs," Water Resources Management, vol. 29, no. 2, pp. 445-457, 2015.

[26] C.-T. Tseng, Y.-L. Lee, and C.-C. Chou, "An ambient intelligence system for water release scheduling in cascade hydropower systems," Journal of Ambient Intelligence and Humanized Computing, vol. 6, no. 1, pp. 47-55, 2015.

[27] Z. K. Feng, W. J. Niu, J. Z. Zhou, C. T. Cheng, H. Qin, and Z. Q. Jiang, "Parallel multi-objective genetic algorithm for short-term economic environmental hydrothermal scheduling," Energies, vol. 10, no. 2, pp. 1-22, 2017.

[28] W. J. Niu, Z. K. Feng, B. F. Feng, Y. W. Min, C. T. Cheng, and J. Z. Zhou, "Comparison of multiple linear regression, artificial neural network, extreme learning machine, and support vector machine in deriving operation rule of hydropower reservoir," Water, vol. 11, pp. 1-17, 2019.

[29] Q. Mao, S. F. Mueller, and H.-M. H. Juang, "Quantitative precipitation forecasting for the Tennessee and cumberland river watersheds using the NCEP regional spectral model," Weather and Forecasting, vol. 15, no. 1, pp. 29-45, 2000.

[30] C. E. M. Tucci, R. T. Clarke, W. Collischonn, P. L. D. S. Dias, and G. S. D. Oliveira, "Long-term flow forecasts based on climate and hydrologic modeling: Uruguay river basin," Water Resources Research, vol. 39, no. 7, p. 1181, 2003.

[31] B. Xu, P.-A. Zhong, R. C. Zambon, Y. Zhao, and W. W.-G. Yeh, "Scenario tree reduction in stochastic programming with recourse for hydropower operations," Water Resources Research, vol. 51, no. 8, pp. 6359-6380, 2015.

[32] S.-E. Fleten and T. K. Kristoffersen, "Short-term hydropower production planning by stochastic programming," Computers \& Operations Research, vol. 35, no. 8, pp. 2656-2671, 2008.

[33] M. Chazarra, J. García-González, J. I. Pérez-Díaz, and M. Arteseros, "Stochastic optimization model for the weekly scheduling of a hydropower system in day-ahead and secondary regulation reserve markets," Electric Power Systems Research, vol. 130, pp. 67-77, 2016.

[34] B. Xu, P.-A. Zhong, Y. Wu, F. Fu, Y. Chen, and Y. Zhao, “A multiobjective stochastic programming model for hydropower hedging operations under inexact information," Water Resources Management, vol. 31, no. 14, pp. 4649-4667, 2017.

[35] S. Séguin, S.-E. Fleten, P. Côté, A. Pichler, and C. Audet, "Stochastic short-term hydropower planning with inflow scenario trees," European Journal of Operational Research, vol. 259, no. 3, pp. 1156-1168, 2017.

[36] J. R. Birge and F. Loveaux, Introduction to Stochastic Programming, Springer-Verlag, Berlin, Germany, 2nd edition, 2011.

[37] M. N. Hjelmeland, J. Zou, A. Helseth, and S. Ahmed, "Nonconvex medium-term hydropower scheduling by stochastic dual dynamic integer programming," IEEE Transactions on Sustainable Energy, vol. 10, no. 1, pp. 481-490, 2018.

[38] A. Helseth, M. Fodstad, and B. Mo, "Optimal medium-term hydropower scheduling considering energy and reserve capacity markets," IEEE Transactions on Sustainable Energy, vol. 7, no. 3, pp. 934-942, 2016.
[39] M. N. Hjelmeland, A. Helseth, and M. Korpås, "Medium-term hydropower scheduling with variable head under inflow, energy and reserve capacity price uncertainty," Energies, vol. 12, pp. 1-15, 2019.

[40] H. Poorsepahy-Samian, V. Espanmanesh, and B. Zahraie, "Improved inflow modeling in stochastic dual dynamic programming," Journal of Water Resources Planning and Management, vol. 142, no. 12, Article ID 04016065, 2016.

[41] Z.-K. Feng, W.-J. Niu, and C.-T. Cheng, "China's large-scale hydropower system: operation characteristics, modeling challenge and dimensionality reduction possibilities," Renewable Energy, vol. 136, pp. 805-818, 2019.

[42] R. C. Grinold, "Model building techniques for the correction of end effects in multistage convex programs," Operations Research, vol. 31, no. 3, pp. 407-431, 1983.

[43] A. J. King and S. W. Wallace, Modeling with Stochastic Programming, Springer International Publishing, New York, USA, 2012.

[44] A. K. Konicz, D. Pisinger, K. M. Rasmussen, and M. Steffensen, "A combined stochastic programming and optimal control approach to personal finance and pensions," OR Spectrum, vol. 37, no. 3, pp. 583-616, 2015.

[45] M. L. Wang, X. Y. Wan, P. A. Zhong, Y. Zhang, and C. Liu, "Characteristics of precipitation in upper reaches of the Yangtze river and its temporal and spatial evolution," Southto-North Water Transfers and Water Science \& Technology, vol. 14, no. 4, pp. 65-71, 2016.

[46] M. Zhang, C. J. Li, Y. C. Zhang, and X. H. Yuan, "Application of minimum risk control model to hydropower generation scheduling," Journal of Huazhong University of Science and Technology (Natural Science Edition), vol. 36, no. 9, pp. 25-28, 2008.

[47] Y. Wang, K. L. Xin, P. Zhao, and J. R. Zhou, "Prediction of seasonal water consumption by Winters method," Water and Wastewater Engineering, vol. 37, no. s1, pp. 183-186, 2011.

[48] Y. D. Yang and Y. Z. Zhang, "On water use situation in the Yangtze river basin," Yangtze River, vol. 37, no. 8, pp. 10-11, 2006. 


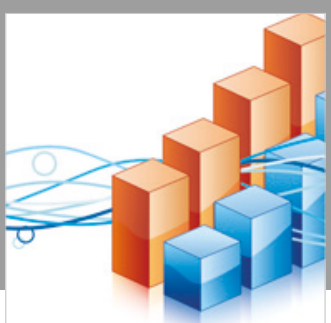

Advances in

Operations Research

\section{-n-m}
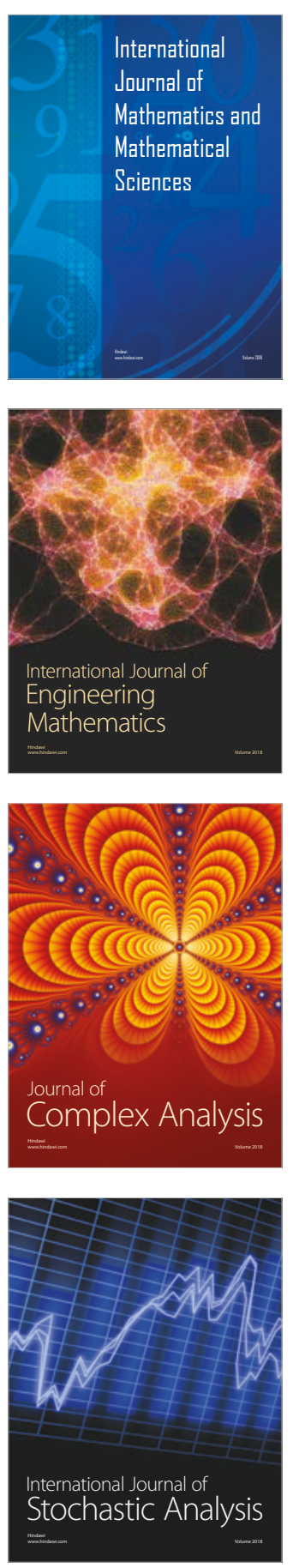
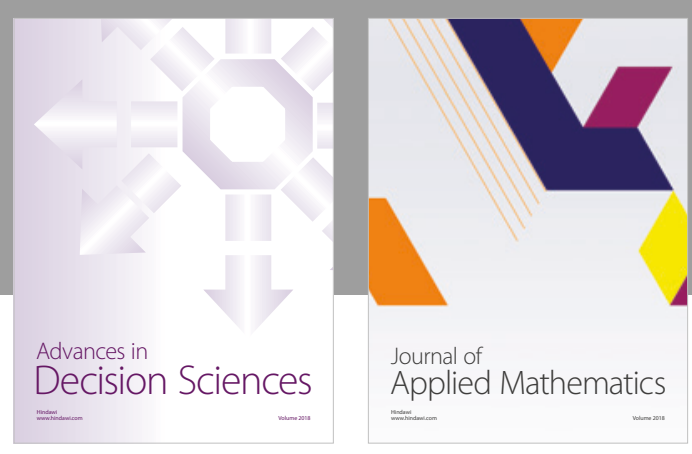

Journal of

Applied Mathematics
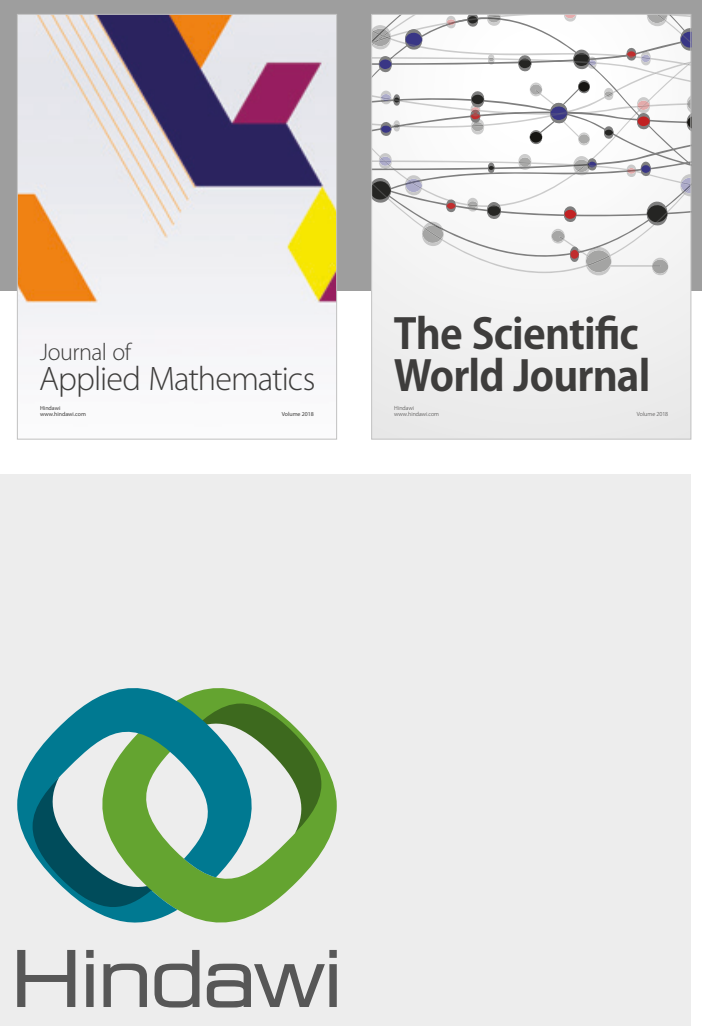

Submit your manuscripts at

www.hindawi.com

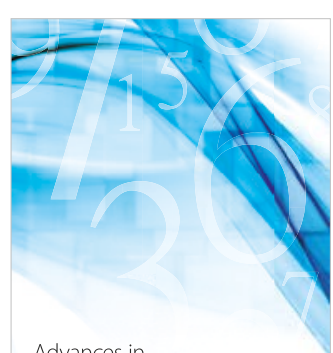

Advances in
Numerical Analysis
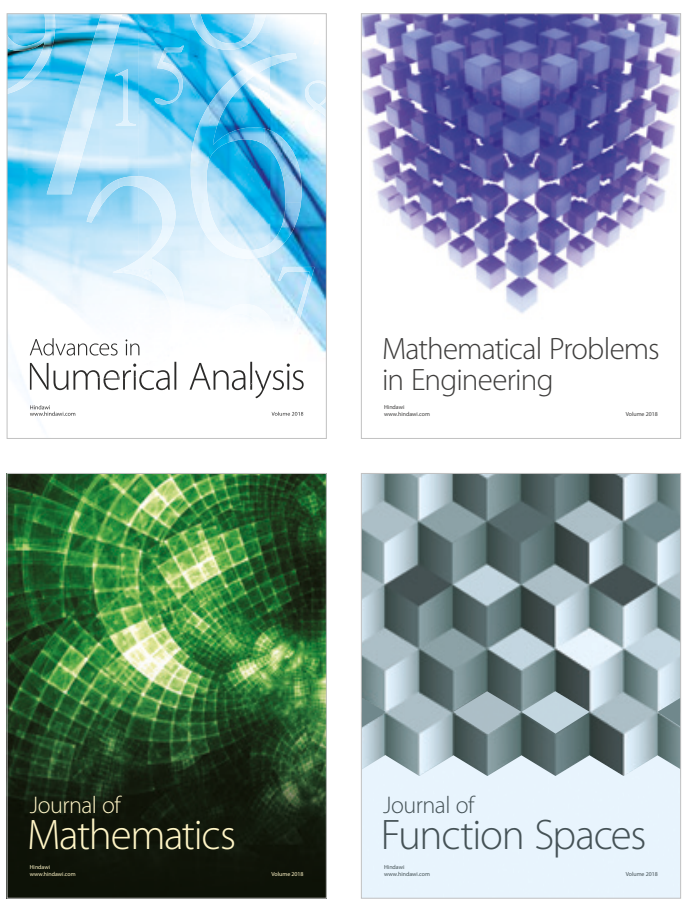

Mathematical Problems in Engineering

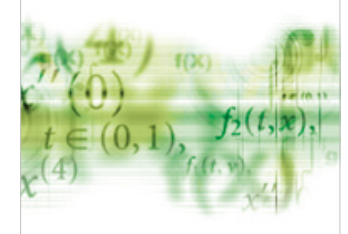

International Journal of

Differential Equations

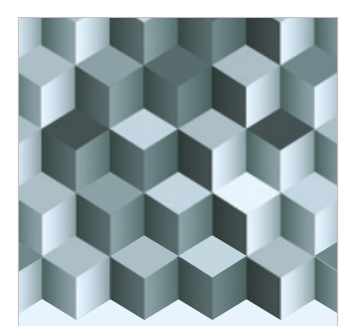

Journal of

Function Spaces
The Scientific

World Journal

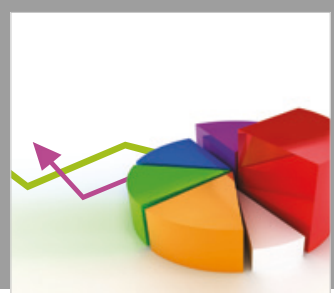

Journal of

Probability and Statistics
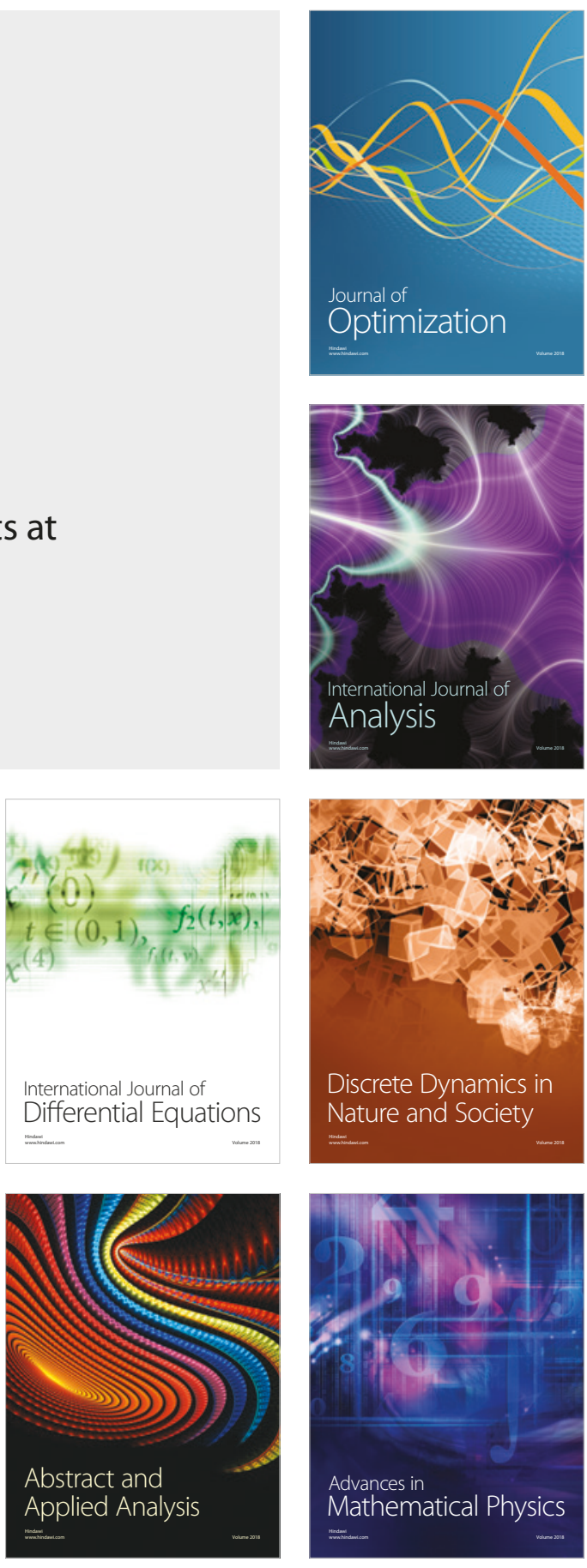